\title{
Calmness and Calculus: Two Basic Patterns
}

\author{
Matúš Benko ${ }^{1}$ (D) Patrick Mehlitz ${ }^{2}$
}

Received: 17 August 2020 / Accepted: 10 May 2021 / Published online: 7 June 2021

(C) The Author(s) 2021

\begin{abstract}
We establish two types of estimates for generalized derivatives of set-valued mappings which carry the essence of two basic patterns observed throughout the pile of calculus rules. These estimates also illustrate the role of the essential assumptions that accompany these two patters, namely calmness on the one hand and (fuzzy) inner calmness* on the other. Afterwards, we study the relationship between and sufficient conditions for the various notions of (inner) calmness. The aforementioned estimates are applied in order to recover several prominent calculus rules for tangents and normals as well as generalized derivatives of marginal functions and compositions as well as Cartesian products of setvalued mappings under mild conditions. We believe that our enhanced approach puts the overall generalized calculus into some other light. Some applications of our findings are presented which exemplary address necessary optimality conditions for minimax optimization problems as well as the calculus related to the recently introduced semismoothness* property.
\end{abstract}

Keywords Calculus · Calmness · Generalized differentiation · Inner calmness* . Set-valued analysis · Variation analysis

Mathematics Subject Classification (2010) 49J52 - 49J53 · 90C31

\section{Introduction}

Mathematical analysis as a whole revolves around the invention of differentiation, which has been gradually extended to plenty of more intricate environments, such as (possibly infinite-dimensional) spaces of various topological structure (Banach spaces, locally convex spaces, etc.). On the other hand, even finite-dimensional optimization, besides numerous

Matúš Benko

matus.benko@univie.ac.at

Patrick Mehlitz

mehlitz@b-tu.de

1 Applied Mathematics and Optimization, University of Vienna, 1090 Vienna, Austria

2 Institute of Mathematics, Brandenburgische Technische Universität Cottbus-Senftenberg, 03046 Cottbus, Germany 
more involved areas of interest, clearly shows that the objects which have to be analyzed are not restricted to single-valued smooth mappings. The need to extend differentiation beyond this setting is, arguably, most successfully implemented by convex analysis, particularly, by the convex subdifferential, see the standard monograph of Rockafellar [45]. Nevertheless, the convex framework is also too limited and not suitable at times, as can be seen, for instance, when equilibrium problems are under consideration. This gives rise to what we call generalized differentiation, which requires no convexity at all.

In order to be able to apply such generalized differentiation in a reasonable manner, basic calculus rules for dealing with, e.g., compositions and sums of functions should be available. Perhaps the most natural extension of the convex subdifferential, the so-called regular (or Fréchet) subdifferential, however, does not obey suitable rules, see [42], and the same applies to its primal counterpart, the so-called subderivative. Among many attempts to overcome this, one of the most prominent has been achieved by Mordukhovich [39, Section 4], who introduced the limiting subdifferential which comes with full calculus at hand.

Ever since, the limiting subdifferential as well as the related normal cone and the associated coderivative have occupied a central place of generalized differentiation and modern variational analysis. In particular, the limiting notions play a crucial role in designing suitable necessary optimality conditions as well as in the characterization of Lipschitzian behavior of set-valued mappings. Many prominent researchers have contributed to their development, and we refer to the two recent monographs of Ioffe [28] and Mordukhovich [41] for detailed information as well as to [40, Section 1.4] for a brief sketch visualizing the historical development of generalized differentiation. Using the recently introduced directional limiting constructions, see [16, 23], the underlying analysis can be refined significantly, as demonstrated by numerous works authored or co-authored by Gfrerer [6, 7, 17-20].

Speaking of calculus rules, we address not only the results concerning subderivatives and subdifferentials of functions, but also the parallel results for tangents and normals to sets on the one hand, and those for graphical derivatives and coderivatives of set-valued mappings on the other. Throughout the whole calculus, however, we observe that most of the rules follow one (or both) of two basic patterns. Moreover, each of these two calculus patterns comes with a characteristic essential assumption given by a certain (Lipschitz) continuity-type requirement for a suitable surrogate set-valued mapping.

The first pattern, represented e.g. by the intersection rule, is more prominent and, thus, also much more studied. In the monographs [40, 46], the essential assumption for this pattern is the so-called Aubin property, which is perhaps the most famous extension of Lipschitz continuity to set-valued mappings and which can be expressed via the so-called Mordukhovich criterion, see Section 4 for details. Later it has been discovered that the Aubin property can be relaxed to calmness, see [25, 26, 29], which is an outer Lipschitzian concept in its nature, related to outer semicontinuity. We would like to mention [19, Proposition 4.1], which presents a rather general, calculus-like estimate. It seems that this result contains the essence of this pattern and that it demonstrates the role of calmness best. Indeed, unlike the standard calculus rules, where the focus is on the estimate and calmness is just a tool that enables it, [19, Proposition 4.1] answers the opposite question, namely: "Having a calm mapping, what can be said about its generalized derivatives?" Note that [19, Proposition 4.1] in fact deals with metric subregularity, which is the "inverse equivalent" of calmness, just like metric regularity is the inverse equivalent of the Aubin property, see [14, Chapter 3]. For more information about calmness and metric subregularity, we further refer the reader to $[13,27,28,41]$. 
The second pattern, represented e.g. by the sum rule (for sets), is certainly less developed. Here, it turns out that the essential role is played by conditions of inner-type. Indeed, the estimates for the limiting constructions are known to hold under inner semicontinuity and inner semicompactness, see [30, 40]. Moreover, the estimates for primal and directional limiting objects were recently shown to hold under inner calmness and inner calmness* in $[4,6]$. While inner calmness* was newly defined in [4], inner calmness can be found under other names, such as, e.g., Lipschitz lower semicontinuity, see [33, 34], or recession with linear rate, see [8, 28], as well. We refer to [8] for a comprehensive overview of this and related notions. Let us also mention the stronger concept of Lipschitz lower semicontinuity*, recently introduced in [9] and motivated by a relaxation of the Aubin property from earlier works of Klatte [31, 32]. We point out that calmness as well as inner calmness* are automatically satisfied by polyhedral set-valued mappings, see [44, Proposition 1] and [4, Theorem 3.4].

The aim of this paper is two-fold. First, we want to establish an inner-type analogue of [19, Proposition 4.1] and then show how these two results, proposed in Theorems 3.1 and 3.2, translate into several standard calculus rules. Particularly, the chain rule for set-valued mappings demonstrates this nicely since both patterns are used for its derivation. We always consider the primal construction (based on tangents) as well as the three dual ones (based on regular, limiting, and directional limiting normals), and we even obtain some new estimates for primal as well as regular dual objects under fuzzy inner calmness*, a relaxation of inner calmness*. The second purpose of the paper is to clarify the relations between the various concepts of (inner) calmness we are going to exploit in our analysis. Particularly, we focus on verifiable sufficient conditions for these properties. Alongside the Aubin property, we utilize the isolated calmness property and the first-order sufficient condition for calmness (FOSCclm for short). These three conditions can be neatly expressed via generalized derivatives, namely the limiting coderivative, graphical derivative, and directional limiting coderivative, respectively. The inverse equivalents of isolated calmness and FOSCclm are strong metric subregularity, see [14], and Gfrerer's first-order sufficient condition for metric subregularity (FOSCMS for short), respectively, see [16], [18, Corollary 1], and [19, Section 2.2]. We note that sufficient conditions for calmness or metric subregularity have been intensively studied in the literature, see e.g. [5, 15, 25, 26, 29, 47]. For calmness notions of inner-type, the obtained results are new. Particularly, in Theorem 4.1, we show that fuzzy inner calmness* is in fact equivalent to validity of the corresponding calculus estimate for primal objects.

The previous discussion suggests that our findings have the potential to be useful in numerous situations. Nevertheless, we only detail two interesting applications. The first one shows how the new rules regarding the regular normal cone can be utilized to infer optimality conditions for hierarchical optimization problems of minimax-type, see [10, 12]. Similarly, these estimates can be used for the derivation of new optimality conditions for equilibrium-constrained mathematical programs, see e.g. [1, 21, 22]. The second example employs the estimates for directional limiting coderivatives to study the newly introduced property of semismoothness* which is instrumental in the construction of Newton-type methods for the numerical solution of generalized equations, see [20].

The remainder of the manuscript is organized as follows: In Section 2, we recall the underlying concepts from set-valued and variational analysis which are utilized in the paper. Section 3 is dedicated to the conception of the two aforementioned calculus patterns. Afterwards, we study the relationship between the various exploited calmness-type conditions and sufficient conditions for their validity in Section 4 . Section 5 provides a collection of 
standard calculus rules, where we elaborate on Cartesian products of set-valued mappings in more detail. Section 6 deals with the aforementioned applications. The paper closes with the aid of some concluding remarks in Section 7.

\section{Preliminaries}

In this section, we provide the essentials of variational analysis and generalized differentiation which can be found in the monographs [3, 40, 41, 46] and the paper [6].

\subsection{Basic Concepts from Variational Analysis}

We use $\mathbb{N}$ and $\mathbb{R}$ to denote the sets of natural and real numbers, respectively. Throughout the paper, we equip $\mathbb{R}^{n}, n \in \mathbb{N}$, with the Euclidean inner product $\langle\cdot, \cdot\rangle: \mathbb{R}^{n} \times \mathbb{R}^{n} \rightarrow \mathbb{R}$ and the Euclidean norm $\|\cdot\|: \mathbb{R}^{n} \rightarrow \mathbb{R}$. The associated unit ball and unit sphere will be denoted by $\mathcal{B}:=\left\{z \in \mathbb{R}^{n} \mid\|z\| \leq 1\right\}$ and $\mathcal{S}:=\left\{z \in \mathbb{R}^{n} \mid\|z\|=1\right\}$, respectively. For a non-empty set $\Omega \subset \mathbb{R}^{n}$, we exploit

$$
\Omega^{\circ}:=\left\{z^{*} \in \mathbb{R}^{n} \mid\left\langle z^{*}, z\right\rangle \leq 0 \forall z \in \Omega\right\}
$$

in order to represent the polar cone of $\Omega$ which is always a closed, convex cone. For arbitrary $\bar{z} \in \mathbb{R}^{n}$, we set $\bar{z}+\Omega=\Omega+\bar{z}:=\{\bar{z}+z \mid z \in \Omega\}$ for brevity of notation. The distance function $\operatorname{dist}(\cdot, \Omega): \mathbb{R}^{n} \rightarrow \mathbb{R}$ of the set $\Omega$ is given by

$$
\operatorname{dist}(z, \Omega):=\inf _{z^{\prime} \in \Omega}\left\|z-z^{\prime}\right\| \quad \forall z \in \mathbb{R}^{n} .
$$

Let $\Omega \subset \mathbb{R}^{n}$ be a set which is locally closed around $\bar{z} \in \Omega$. The tangent (or Bouligand, contingent) cone to $\Omega$ at $\bar{z}$ is given by

$$
T_{\Omega}(\bar{z}):=\left\{w \in \mathbb{R}^{n} \mid \exists\left(t_{k}\right) \downarrow 0, \exists\left(w_{k}\right) \rightarrow w: \bar{z}+t_{k} w_{k} \in \Omega \forall k \in \mathbb{N}\right\} .
$$

Furthermore, we use

$$
\widehat{N}_{\Omega}(\bar{z}):=T_{\Omega}(\bar{z})^{\circ}
$$

in order to represent the so-called regular (or Fréchet) normal cone to $\Omega$ at $\bar{z}$. We refer to

$$
N_{\Omega}(\bar{z}):=\left\{z^{*} \in \mathbb{R}^{n} \mid \exists\left(z_{k}\right) \rightarrow \bar{z}, \exists\left(z_{k}^{*}\right) \rightarrow z^{*}: z_{k}^{*} \in \widehat{N}_{\Omega}\left(z_{k}\right) \forall k \in \mathbb{N}\right\}
$$

as the limiting (or Mordukhovich) normal cone to $\Omega$ at $\bar{z}$. By definition, $\widehat{N}_{\Omega}(\bar{z}) \subset N_{\Omega}(\bar{z})$ holds. In case where $\Omega$ is convex, we have the relations

$$
\widehat{N}_{\Omega}(\bar{z})=N_{\Omega}(\bar{z})=(\Omega-\bar{z})^{\circ},
$$

i.e., the regular and limiting normal cone to $\Omega$ at $\bar{z}$ amount to the classical normal cone in the sense of convex analysis.

Finally, given a direction $w \in \mathbb{R}^{n}$, we denote by

$$
N_{\Omega}(\bar{z} ; w):=\left\{z^{*} \in \mathbb{R}^{n} \mid \exists\left(t_{k}\right) \downarrow 0, \exists\left(w_{k}\right) \rightarrow w, \exists\left(z_{k}^{*}\right) \rightarrow z^{*}: z_{k}^{*} \in \widehat{N}_{\Omega}\left(\bar{z}+t_{k} w_{k}\right) \forall k \in \mathbb{N}\right\}
$$

the directional limiting normal cone to $\Omega$ at $\bar{z}$ in direction $w$. This comparatively new variational object has been introduced in [16, 23]. In [6], main calculus rules for directional limiting normals are discussed, see [4, Section 4] and [47, Section 3] as well. Amongst others, let us mention that $N_{\Omega}(\bar{z} ; w)=\varnothing$ holds for all $w \notin T_{\Omega}(\bar{z})$. Moreover, we have

$$
N_{\Omega}(\bar{z})=\widehat{N}_{\Omega}(\bar{z}) \cup \bigcup_{w \in T_{\Omega}(\bar{z}) \cap \mathcal{S}} N_{\Omega}(\bar{z} ; w),
$$

and $N_{\Omega}(\bar{z} ; 0)=N_{\Omega}(\bar{z})$ is obvious. 
For each $\tilde{z} \notin \Omega$ and arbitrary $w \in \mathbb{R}^{n}$, we set $T_{\Omega}(\tilde{z}):=\varnothing$ as well as $\widehat{N}_{\Omega}(\tilde{z})=N_{\Omega}(\tilde{z})=$ $N_{\Omega}(\tilde{z} ; w):=\varnothing$ for completeness.

Let us now mention two elementary results for the calculus of tangents and normals addressing changes of coordinates and product structures.

Lemma 2.1 Let $g: \mathbb{R}^{n} \rightarrow \mathbb{R}^{m}$ be a continuously differentiable function and let $C \subset \mathbb{R}^{m}$ be locally closed around $g(x) \in C$ for some fixed $x \in \mathbb{R}^{n}$. Finally, assume that the Jacobian $\nabla g(x)$ possesses full row rank $m$. Then we have

$$
\begin{gathered}
T_{g^{-1}(C)}(x) \quad=\left\{u \mid \nabla g(x) u \in T_{C}(g(x))\right\}, \\
\widehat{N}_{g^{-1}(C)}(x) \quad=\left\{\nabla g(x)^{\top} y \mid y \in \widehat{N}_{C}(g(x))\right\}, \\
N_{g^{-1}(C)}(x) \quad=\left\{\nabla g(x)^{\top} y \mid y \in N_{C}(g(x))\right\}, \\
N_{g^{-1}(C)}(x ; u)=\left\{\nabla g(x)^{\top} y \mid y \in N_{C}(g(x) ; \nabla g(x) u)\right\} .
\end{gathered}
$$

Proof The formulas for tangents as well as regular and limiting normals follow from [46, Exercise 6.7]. It remains to verify the formula for directional limiting normals.

In case $n=m$, the regularity of $\nabla g(x)$ extends to a neighborhood $U$ of $x$ where these matrices are continuously invertible. Using now the available formula for the regular normal cone to $g^{-1}(C)$ for all points from $g^{-1}(C) \cap U$, we easily infer the desired result from the definition of the directional limiting normal cone. In case $n>m$, we exploit the trick from [46, Exercise 6.7] in order to deduce the more general statement from the former arguments.

Lemma 2.2 For natural numbers $n_{i} \in \mathbb{N}, i=1, \ldots, \ell$, sets $C_{i} \subset \mathbb{R}^{n_{i}}$, points $x_{i} \in C_{i}$ where $C_{i}$ is locally closed, and directions $u_{i} \in \mathbb{R}^{n_{i}}$, we have

$$
\begin{aligned}
T_{\prod_{i=1}^{\ell} C_{i}}\left(x_{1}, \ldots, x_{\ell}\right) & \subset \prod_{i=1}^{\ell} T_{C_{i}}\left(x_{i}\right), \\
\widehat{N}_{\prod_{i=1}^{\ell} C_{i}}\left(x_{1}, \ldots, x_{\ell}\right) & =\prod_{i=1}^{\ell} \widehat{N}_{C_{i}}\left(x_{i}\right), \\
N_{\prod_{i=1}^{\ell} C_{i}}\left(x_{1}, \ldots, x_{\ell}\right) & =\prod_{i=1}^{\ell} N_{C_{i}}\left(x_{i}\right), \\
N_{\prod_{i=1}^{\ell} C_{i}}\left(\left(x_{1}, \ldots, x_{\ell}\right) ;\left(u_{1}, \ldots, u_{\ell}\right)\right) & \subset \prod_{i=1}^{\ell} N_{C_{i}}\left(x_{i} ; u_{i}\right) .
\end{aligned}
$$

Proof The formulas for regular and limiting normals are stated in [46, Proposition 6.41]. For the remaining inclusions, we refer the reader to [47, Proposition 3.3].

In case where the sets under consideration possess additional regularity properties, it is even possible to obtain equality in the formulas for tangents and directional limiting normals in Lemma 2.2, see [47, Section 3]. Particularly, let us mention that equality is obtained in these formulas if the product of two sets is considered and one of them is convex or equals the graph of a continuously differentiable mapping.

Next, we recall the basic notions of generalized differentiation. For a set-valued mapping $M: \mathbb{R}^{m} \rightrightarrows \mathbb{R}^{n}, \operatorname{dom} M:=\left\{y \in \mathbb{R}^{m} \mid M(y) \neq \varnothing\right\}$ and gph $M:=\left\{(y, x) \in \mathbb{R}^{m} \times \mathbb{R}^{n} \mid x \in\right.$ $M(y)\}$ denote the domain and the graph of $M$, respectively. The mapping $M^{-1}: \mathbb{R}^{n} \rightrightarrows \mathbb{R}^{m}$ given by $M^{-1}(x):=\left\{y \in \mathbb{R}^{m} \mid x \in M(y)\right\}$ for all $x \in \mathbb{R}^{n}$ is referred to as the inverse of $M$.

Let $(\bar{y}, \bar{x}) \in \operatorname{gph} M$ be a point where gph $M$ is locally closed. In this paper, we will consider four types of generalized derivatives of $M$ corresponding to the four different cones 
from above applied to gph $M$ at $(\bar{y}, \bar{x})$. The set-valued mapping $D M(\bar{y}, \bar{x}): \mathbb{R}^{m} \rightrightarrows \mathbb{R}^{n}$, defined by

$$
D M(\bar{y}, \bar{x})(v):=\left\{u \in \mathbb{R}^{n} \mid(v, u) \in T_{\operatorname{gph} M}(\bar{y}, \bar{x})\right\} \quad \forall v \in \mathbb{R}^{m},
$$

is called the graphical (or Bouligand) derivative of $M$ at $(\bar{y}, \bar{x})$. We refer to the mapping $\widehat{D}^{*} M(\bar{y}, \bar{x}): \mathbb{R}^{n} \rightrightarrows \mathbb{R}^{m}$, given by

$$
\widehat{D}^{*} M(\bar{y}, \bar{x})\left(x^{*}\right):=\left\{y^{*} \in \mathbb{R}^{m} \mid\left(y^{*},-x^{*}\right) \in \widehat{N}_{\mathrm{gph} M}(\bar{y}, \bar{x})\right\} \quad \forall x^{*} \in \mathbb{R}^{n},
$$

as the regular (or Fréchet) coderivative of $M$ at $(\bar{y}, \bar{x})$. The mapping $D^{*} M(\bar{y}, \bar{x}): \mathbb{R}^{n} \rightrightarrows$ $\mathbb{R}^{m}$, defined via

$$
D^{*} M(\bar{y}, \bar{x})\left(x^{*}\right):=\left\{y^{*} \in \mathbb{R}^{m} \mid\left(y^{*},-x^{*}\right) \in N_{\mathrm{gph} M}(\bar{y}, \bar{x})\right\} \quad \forall x^{*} \in \mathbb{R}^{n},
$$

is referred to as the limiting (or Mordukhovich) coderivative of $M$ at $(\bar{y}, \bar{x})$. Finally, given a pair of directions $(v, u) \in \mathbb{R}^{m} \times \mathbb{R}^{n}$, the mapping $D^{*} M((\bar{y}, \bar{x}) ;(v, u)): \mathbb{R}^{n} \rightrightarrows \mathbb{R}^{m}$, given by

$$
D^{*} M((\bar{y}, \bar{x}) ;(v, u))\left(x^{*}\right):=\left\{y^{*} \in \mathbb{R}^{m} \mid\left(y^{*},-x^{*}\right) \in N_{\operatorname{gph} M}((\bar{y}, \bar{x}) ;(v, u))\right\} \quad \forall x^{*} \in \mathbb{R}^{n},
$$

is called the directional limiting coderivative of $M$ in direction $(v, u)$ at $(\bar{y}, \bar{x})$. For a singlevalued mapping $F: \mathbb{R}^{m} \rightarrow \mathbb{R}^{n}$ and some point $\bar{y} \in \mathbb{R}^{m}$, we denote the above derivatives without $\bar{x}=F(\bar{y})$ for brevity. In case where $F$ is continuously differentiable at $\bar{y}$, we obtain the relations $D F(\bar{y})(v)=\nabla F(\bar{y}) v$ as well as $\widehat{D}^{*} F(\bar{y})\left(x^{*}\right)=D^{*} F(\bar{y})\left(x^{*}\right)=\nabla F(\bar{y})^{\top} x^{*}$ for arbitrary $v \in \mathbb{R}^{m}$ and $x^{*} \in \mathbb{R}^{n}$. Particularly, we have $D^{*} F(\bar{y} ;(v, u))\left(x^{*}\right) \neq \varnothing$ if and only if $u=\nabla F(\bar{y}) v$ holds. In this case, $D^{*} F(\bar{y} ;(v, u))\left(x^{*}\right)=\nabla F(\bar{y})^{\top} x^{*}$ follows.

For an extended real-valued function $f: \mathbb{R}^{n} \rightarrow \overline{\mathbb{R}}$, epi $f:=\left\{(x, \alpha) \in \mathbb{R}^{n} \times \mathbb{R} \mid f(x) \leq\right.$ $\alpha\}$ denotes its epigraph. It is well known that $f$ is lower semicontinuous at some point $\bar{x} \in \mathbb{R}^{n}$ satisfying $|f(\bar{x})|<\infty$ if and only if epi $f$ is locally closed at $(\bar{x}, f(\bar{x}))$. Fix such a point $\bar{x} \in \mathbb{R}^{n}$. For the purposes of this paper, we avoid the standard definitions of generalized derivatives of $f$ but introduce them rather via their characterizations in terms of tangents and normals to epi $f$. The extended real-valued function $\mathrm{d} f(\bar{x}): \mathbb{R}^{n} \rightarrow \overline{\mathbb{R}}$, characterized by

$$
\text { epi } \mathrm{d} f(\bar{x})=T_{\text {epi } f}(\bar{x}, f(\bar{x})) \text {, }
$$

is called the subderivative of $f$ at $\bar{x}$. Furthermore, we define the regular and limiting subdifferential of $f$ at $\bar{x}$ by means of

$$
\begin{aligned}
& \widehat{\partial} f(\bar{x}):=\left\{x^{*} \in \mathbb{R}^{n} \mid\left(x^{*},-1\right) \in \widehat{N}_{\text {epi } f(\bar{x}, f(\bar{x}))\},}\right. \\
& \partial f(\bar{x}):=\left\{x^{*} \in \mathbb{R}^{n} \mid\left(x^{*},-1\right) \in N_{\text {epi } f}(\bar{x}, f(\bar{x}))\right\},
\end{aligned}
$$

respectively. Finally, for each pair $(u, \mu) \in \mathbb{R}^{n} \times \mathbb{R}$, we refer to

$$
\begin{aligned}
\partial f(\bar{x} ;(u, \mu)) & :=\left\{x^{*} \in \mathbb{R}^{n} \mid\left(x^{*},-1\right) \in N_{\text {epi } f}((\bar{x}, f(\bar{x})) ;(u, \mu))\right\}, \\
\partial^{\infty} f(\bar{x} ;(u, \mu)) & :=\left\{x^{*} \in \mathbb{R}^{n} \mid\left(x^{*}, 0\right) \in N_{\text {epi } f}((\bar{x}, f(\bar{x})) ;(u, \mu))\right\}
\end{aligned}
$$

as the directional limiting subdifferential of $f$ at $\bar{x}$ in direction $(u, \mu)$ and the singular directional limiting subdifferential of $f$ at $\bar{x}$ in direction $(u, \mu)$, respectively. For a connection to generalized derivatives of set-valued mappings, we refer to [46, Theorem 8.2].

\subsection{Stability Notions for Set-Valued Mappings}

Below, we present the definitions of the most important stability concepts for this paper. We note that several other related notions are introduced and studied in Section 4. Recall that a sequence $\left(z_{k}\right)$ is said to converge to $z$ from a direction $w$ whenever there are sequences $\left(t_{k}\right) \downarrow 0$ and $\left(w_{k}\right) \rightarrow w$ such that $z_{k}=z+t_{k} w_{k}$ holds for all $k \in \mathbb{N}$. 
We start by recalling the definitions of inner semicompactness and inner calmness*.

Definition 2.3 Let $M: \mathbb{R}^{m} \rightrightarrows \mathbb{R}^{n}$ be a set-valued mapping and fix $\bar{y} \in \operatorname{dom} M$. Furthermore, let $\Omega \subset \mathbb{R}^{m}$ and as well as a direction $v \in \mathbb{R}^{m}$ be arbitrarily chosen.

(i) We say that $M$ is inner semicompact at $\bar{y}$ w.r.t. $\Omega$ if for each sequence $\left(y_{k}\right) \subset \Omega$ converging to $\bar{y}$, we find $\bar{x} \in \mathbb{R}^{n}$ and a sequence $\left(x_{k}\right)$ such that $\left(x_{k}\right) \rightarrow \bar{x}$ and $x_{k} \in M\left(y_{k}\right)$ hold along a subsequence. In case where this property only holds for all sequences $\left(y_{k}\right)$ converging to $\bar{y}$ from $v$, we call $M$ inner semicompact at $\bar{y}$ w.r.t. $\Omega$ in direction $v$.

(ii) We say that $M$ is inner calm* at $\bar{y}$ w.r.t. $\Omega$ if there is some $\kappa>0$ such that for each sequence $\left(y_{k}\right) \subset \Omega$ converging to $\bar{y}$, we find $\bar{x} \in \mathbb{R}^{n}$ and a sequence $\left(x_{k}\right)$ such that $\left\|x_{k}-\bar{x}\right\| \leq \kappa\left\|y_{k}-\bar{y}\right\|$ and $x_{k} \in M\left(y_{k}\right)$ hold along a subsequence. The infimum $\bar{\kappa}$ over all constants with this property is called modulus of inner calmness* at $\bar{y}$ w.r.t. $\Omega$. In case where the above property only holds for all sequences $\left(y_{k}\right)$ converging to $\bar{y}$ from $v$, we call $M$ inner calm* at $\bar{y}$ w.r.t. $\Omega$ in direction $v$. Similarly as above, we introduce the modulus $\bar{\kappa}_{v}$ of inner calmness* at $\bar{y}$ w.r.t. $\Omega$ in direction $v$.

In case where $\Omega:=\mathbb{R}^{m}$ can be chosen, we simply omit the expression "w.r.t. $\mathbb{R}^{m}$ " for brevity.

Clearly, inner calmness* of a set-valued mapping at one point of its domain is stronger than inner semicompactness since the former provides an additional information about the rate of convergence. Note that whenever $M: \mathbb{R}^{m} \rightrightarrows \mathbb{R}^{n}$ is a set-valued mapping with a closed graph, then $\operatorname{dom} M$ does not need to be closed. However, fixing $\bar{y} \in \operatorname{dom} M$ where $M$ is inner semicompact w.r.t. $\operatorname{dom} M$ yields closedness of $\operatorname{dom} M$ locally around $\bar{y}$, see [4, Lemma 2.1].

Next, we present the definition of a relaxed version inner calmness*.

Definition 2.4 Let $M: \mathbb{R}^{m} \rightrightarrows \mathbb{R}^{n}$ be a set-valued mapping and fix $\bar{y} \in \operatorname{dom} M$, a set $\Omega \subset \mathbb{R}^{m}$ which is locally closed at $\bar{y}$, and a direction $v \in \mathbb{R}^{m}$. We say that $M$ is inner calm* in the fuzzy sense at $\bar{y}$ w.r.t. $\Omega$ in direction $v$ if either $v \notin T_{\Omega}(\bar{y})$ holds or if there is some $\kappa_{v}>0$ such that we find a sequence $\left(y_{k}\right) \subset \Omega$ converging to $\bar{y}$ from $v$, some $\bar{x} \in \mathbb{R}^{n}$, and a sequence $\left(x_{k}\right)$ such that $x_{k} \in M\left(y_{k}\right)$ and $\left\|x_{k}-\bar{x}\right\| \leq \kappa_{v}\left\|y_{k}-\bar{y}\right\|$ hold for all $k \in \mathbb{N}$. The infimum $\bar{\kappa}_{v}$ over all constants with this property is called modulus of inner calmness* in the fuzzy sense at $\bar{y}$ w.r.t. $\Omega$ in direction $v$. For $v \notin T_{\Omega}(\bar{y})$, we set $\bar{\kappa}_{v}:=0$ for the purpose of completeness. We say that $M$ is inner calm* in the fuzzy sense at $\bar{y}$ w.r.t. $\Omega$ provided it is inner calm* in the fuzzy sense at $\bar{y}$ w.r.t. $\Omega$ in each direction $v \in \mathcal{S}$. In case where $\Omega:=\mathbb{R}^{m}$ can be chosen, we simply omit the expression "w.r.t. $\mathbb{R}^{m}$ " for brevity.

Inner calmness* and inner calmness* in the fuzzy sense have been first defined in [4]. The motivation for introducing the fuzzy inner calmness* comes from the very natural setting of a certain multiplier mapping which possesses this property, see [4, Theorem 3.9]. It is worth noting that fuzzy inner calmness* does not imply inner semicompactness in general. In this paper, we extend the calculus rules based on (fuzzy) inner calmness* from [4, Section 4], particularly to estimates for regular normals.

Note that each of the "inner" conditions from Definitions 2.3 and 2.4 implies that the images of the underlying set-valued mapping are non-empty for all points of the set $\Omega$ that lie near the point of interest $\bar{y}$. While this can be desirable in some situations, it turns out to be quite restrictive in other settings. For our purposes, however, we will often consider these properties w.r.t. the domain of the mapping, and this does not add any restriction. 
Finally, we would like to recall the definition of calmness.

Definition 2.5 Let $M: \mathbb{R}^{m} \rightrightarrows \mathbb{R}^{n}$ be a set-valued mapping and fix $(\bar{y}, \bar{x}) \in \operatorname{gph} M$ as well as a direction $u \in \mathbb{R}^{n}$. We say that $M$ is calm at $(\bar{y}, \bar{x})$ if there is some $\kappa>0$ such that for each sequence $\left(x_{k}\right) \rightarrow \bar{x}$ satisfying $x_{k} \in M\left(y_{k}\right)$ for some $y_{k}$ and all $k \in \mathbb{N}$, we find a sequence $\left(\tilde{x}_{k}\right) \subset M(\bar{y})$ such that $\left\|x_{k}-\tilde{x}_{k}\right\| \leq \kappa\left\|y_{k}-\bar{y}\right\|$ holds for sufficiently large $k \in \mathbb{N}$. The infimum $\bar{\kappa}$ over all constants with this property is called the modulus of calmness at $(\bar{y}, \bar{x})$. In case where the above property only holds for all sequences $\left(x_{k}\right)$ converging to $\bar{x}$ from $u$, we call $M$ calm at $(\bar{y}, \bar{x})$ in direction $u$. Similarly as above, we introduce the modulus $\bar{\kappa}_{u}$ of calmness at $(\bar{y}, \bar{x})$ in direction $u$.

One can easily check that $M$ is calm at $(\bar{y}, \bar{x}) \in \operatorname{gph} M$ if and only if there are neighborhoods $U$ of $\bar{x}$ and $V$ of $\bar{y}$ as well as some constant $\kappa>0$ such that

$$
M(y) \cap U \subset M(\bar{y})+\kappa\|y-\bar{y}\| \mathcal{B} \quad \forall y \in V
$$

holds, and this corresponds to the classical definition of calmness. In order to be consistent with the definition of inner calmness*, however, we choose to work with the sequential counterpart of the definition in this manuscript. It is well known that the neighborhood $U$ can be reduced in such a way that $V$ can be replaced by the whole space $\mathbb{R}^{m}$, see [14, Exercise 3H.4]. On the one hand, this leads to the equivalent formulation of calmness via

$$
\operatorname{dist}(x, M(\bar{y})) \leq \kappa \operatorname{dist}\left(\bar{y}, M^{-1}(x)\right) \quad \forall x \in U,
$$

which is precisely the definition of metric subregularity of $M^{-1}$ at $(\bar{x}, \bar{y})$. On the other hand, it motivates us to define directional calmness via directions in the range space $\mathbb{R}^{n}$ and, in turn, yields that calmness in a predefined direction $u$ is the inverse counterpart of metric subregularity in direction $u$, see [16].

In Section 4, we relate the properties from above to other prominent Lipschitzian notions.

We conclude this preliminary section with the following simple result regarding the stability of calmness and inner calmness* under single-valued calm perturbations. A singlevalued function $\varphi: \mathbb{R}^{n} \rightarrow \mathbb{R}^{m}$ is called calm at $\bar{x} \in \mathbb{R}^{n}$ if there is a constant $\kappa>0$ such that

$$
\left\|\varphi\left(x_{k}\right)-\varphi(\bar{x})\right\| \leq \kappa\left\|x_{k}-\bar{x}\right\|
$$

holds for every sequence $\left(x_{k}\right) \rightarrow \bar{x}$ and sufficiently large $k \in \mathbb{N}$.

Proposition 2.6 Let $M: \mathbb{R}^{m} \rightrightarrows \mathbb{R}^{n}$ be a set-valued mapping, let $\varphi_{1}: \mathbb{R}^{\ell} \rightarrow \mathbb{R}^{r}, \varphi_{2}: \mathbb{R}^{\ell} \rightarrow$ $\mathbb{R}^{m}$ and $\varphi_{3}: \mathbb{R}^{\ell} \rightarrow \mathbb{R}^{n}$ be single-valued mappings, and define another set-valued mapping $\tilde{M}: \mathbb{R}^{\ell} \rightrightarrows \mathbb{R}^{r} \times \mathbb{R}^{n}$ as follows:

$$
\tilde{M}(q):=\left\{\varphi_{1}(q)\right\} \times\left(M\left(\varphi_{2}(q)\right)+\varphi_{3}(q)\right) \quad \forall q \in \mathbb{R}^{\ell} .
$$

(i) If the functions $\varphi_{i}, i=1,2,3$, are continuous (calm) at $\bar{q}$ and if $M$ is inner semicompact (inner calm ${ }^{*}$ ) at $\varphi_{2}(\bar{q})$ w.r.t. $\operatorname{dom} M$, then $\widetilde{M}$ is inner semicompact (inner calm*) at $\bar{q}$ w.r.t. $\operatorname{dom} \tilde{M}$.

(ii) If the functions $\varphi_{i}, i=1,2,3$, are calm at $\bar{q}$ and if $M$ is calm at $\left(\varphi_{2}(\bar{q}), \bar{x}\right) \in \operatorname{gph} M$ for some $\bar{x}$, then $\widetilde{M}$ is calm at $\left(\bar{q},\left(\varphi_{1}(\bar{q}), \bar{x}+\varphi_{3}(\bar{q})\right)\right) \in \operatorname{gph} \widetilde{M}$.

Proof The proof of the inner semicompactness claim from (i) is simpler, so we only prove the claim regarding inner calmness*. For each $i=1,2,3$, let $\kappa_{i}$ be a calmness constant of $\varphi_{i}$, and let $\kappa>0$ be an inner calmness* constant of $M$ at $\varphi_{2}(\bar{q})$ w.r.t. $\operatorname{dom} M$. Furthermore, let us equip product spaces w.l.o.g. with the natural sum norms. In order to 
prove (i), consider a sequence $\left(q_{k}\right) \rightarrow \bar{q}$ with $\left(q_{k}\right) \subset \operatorname{dom} \tilde{M}$. This automatically yields $\left(\varphi_{2}\left(q_{k}\right)\right) \subset \operatorname{dom} M$. The calmness of $\varphi_{2}$ at $\bar{q}$ guarantees $\varphi\left(q_{k}\right) \rightarrow \varphi(\bar{q})$. Thus, by inner calmness* of $M$, we find a sequence $\left(x_{k}\right)$ and a point $\bar{x}$ such that $x_{k} \in M\left(\varphi_{2}\left(q_{k}\right)\right)$ and $\left\|x_{k}-\bar{x}\right\| \leq \kappa\left\|\varphi_{2}\left(q_{k}\right)-\varphi(\bar{q})\right\|$ hold along a subsequence. Consequently, we find that

$$
\begin{aligned}
& \left\|\left(\varphi_{1}\left(q_{k}\right), x_{k}+\varphi_{3}\left(q_{k}\right)\right)-\left(\varphi_{1}(\bar{q}), \bar{x}+\varphi_{3}(\bar{q})\right)\right\| \\
& \leq\left\|x_{k}-\bar{x}\right\|+\left\|\varphi_{1}\left(q_{k}\right)-\varphi_{1}(\bar{q})\right\|+\left\|\varphi_{3}\left(q_{k}\right)-\varphi_{3}(\bar{q})\right\| \\
& \leq \kappa\left\|\varphi_{2}\left(q_{k}\right)-\varphi_{2}(\bar{q})\right\|+\left(\kappa_{1}+\kappa_{3}\right)\left\|q_{k}-\bar{q}\right\| \\
& \leq\left(\kappa \kappa_{2}+\kappa_{1}+\kappa_{3}\right)\left\|q_{k}-\bar{q}\right\|
\end{aligned}
$$

holds along a subsequence. Since $\left(\varphi_{1}\left(q_{k}\right), x_{k}+\varphi_{3}\left(q_{k}\right)\right) \in \tilde{M}\left(q_{k}\right)$ holds along a subsequence, the claim follows.

Let us now prove (ii). Therefore, let $\kappa>0$ be a calmness constant of $M$ at $\left(\varphi_{2}(\bar{q}), \bar{x}\right)$. Consider a sequence $\left(\left(a_{k}, b_{k}\right)\right) \rightarrow\left(\varphi_{1}(\bar{q}), \bar{x}+\varphi_{3}(\bar{q})\right)$ with $\left(a_{k}, b_{k}\right) \in \widetilde{M}\left(q_{k}\right)$ for some $q_{k}$ and all $k \in \mathbb{N}$, i.e., $\left(a_{k}, b_{k}\right)=\left(\varphi_{1}\left(q_{k}\right), x_{k}+\varphi_{3}\left(q_{k}\right)\right)$ for some $x_{k} \in M\left(\varphi_{2}\left(q_{k}\right)\right)$ and all $k \in \mathbb{N}$ such that $\left(x_{k}\right) \rightarrow \bar{x}$. The calmness of $M$ now yields the existence of a sequence $\left(\tilde{x}_{k}\right) \subset M\left(\varphi_{2}(\bar{q})\right)$ such that the above estimates with $\bar{x}$ replaced by $\tilde{x}_{k}$ hold for sufficiently large $k \in \mathbb{N}$. This completes the proof since $\left(\varphi_{1}(\bar{q}), \tilde{x}_{k}+\varphi_{3}(\bar{q})\right) \in \tilde{M}(\bar{q})$ holds.

\section{On Two Patterns in Calculus}

From Definitions 2.3 to 2.5 , one could perhaps guess that inner calmness* w.r.t. the domain in fact provides a connection between the domain of a set-valued mapping $M: \mathbb{R}^{m} \rightrightarrows \mathbb{R}^{n}$ and its graph, while calmness connects the graph of $M$ with its image sets. Here, we will formalize this conjecture by comparing tangents and normals to the graph with tangents and normals to the domain under the inner calmness* on one hand, and with tangents and normals to the images under the calmness on the other hand. Let us start with the former.

Theorem 3.1 Let $M: \mathbb{R}^{m} \rightrightarrows \mathbb{R}^{n}$ be a set-valued mapping and let $\bar{y} \in \operatorname{dom} M$ be chosen such that gph $M$ is locally closed around $\{\bar{y}\} \times \mathbb{R}^{n}$ and $\operatorname{dom} M$ is locally closed around $\bar{y}$. Then the following assertions hold.

T Tangents: We always have

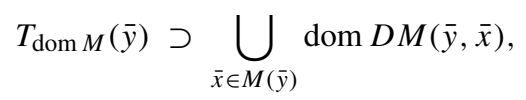

and the opposite inclusion holds true if $M$ is inner calm* at $\bar{y}$ w.r.t. $\operatorname{dom} M$ in the fuzzy sense. Moreover, if $\bar{\kappa}_{v}$ denotes the modulus of inner calmness ${ }^{*}$ of $M$ at $\bar{y}$ w.r.t. $\operatorname{dom} M$ in direction $v$ in the fuzzy sense, we in fact have

$$
T_{\operatorname{dom} M}(\bar{y})=\left\{v \mid \inf _{\bar{x} \in M(\bar{y})} \inf _{u \in D M(\bar{y}, \bar{x})(v)}\|u\| \leq \bar{\kappa}_{v}\|v\|\right\},
$$

and this holds with $\bar{\kappa}_{v}$ replaced by $\bar{\kappa}$ provided $M$ is even inner calm $*$ at $\bar{y}$ w.r.t. $\operatorname{dom} M$ with modulus $\bar{\kappa}$.

$\widehat{\mathbf{N}}$ Regular normals: We always have

$$
\widehat{N}_{\mathrm{dom} M}(\bar{y}) \subset \bigcap_{\bar{x} \in M(\bar{y})} \widehat{D}^{*} M(\bar{y}, \bar{x})(0),
$$


and the opposite inclusion holds true if $M$ is inner calm* at $\bar{y}$ w.r.t. $\operatorname{dom} M$ in the fuzzy sense.

$\mathbf{N}$ Limiting normals: If $M$ is inner semicompact at $\bar{y}$ w.r.t. $\operatorname{dom} M$, then we have

$$
N_{\operatorname{dom} M}(\bar{y}) \subset \bigcup_{\bar{x} \in M(\bar{y})} D^{*} M(\bar{y}, \bar{x})(0) .
$$

dN Directional limiting normals: Let $v \in \mathbb{R}^{m}$ be a fixed direction. If $M$ is inner semicompact at $\bar{y}$ in direction $v$ w.r.t. $\operatorname{dom} M$, then we have

$$
\begin{aligned}
N_{\operatorname{dom} M}(\bar{y} ; v) \subset \bigcup_{\bar{x} \in M(\bar{y})} & {\left[\bigcup_{u \in D M(\bar{y}, \bar{x})(v)} D^{*} M((\bar{y}, \bar{x}) ;(v, u))(0)\right.} \\
\cup & \left.\bigcup \bigcup_{u \in D M(\bar{y}, \bar{x})(0) \cap \mathcal{S}} D^{*} M((\bar{y}, \bar{x}) ;(0, u))(0)\right] .
\end{aligned}
$$

Moreover, the union over $D M(\bar{y}, \bar{x})(0) \cap \mathcal{S}$ is superfluous if $M$ is inner calm* at $\bar{y}$ in direction $v$ w.r.t. $\operatorname{dom} M$ with modulus $\bar{\kappa}_{v}$, and $u \in D M(\bar{y}, \bar{x})(v)$ can be chosen with $\|u\| \leq \kappa\|v\|$ for any $\kappa>\bar{\kappa}_{v}$ in this case.

Proof In order to prove T, suppose first that there exist $\bar{x} \in M(\bar{y})$ and $u \in D M(\bar{y}, \bar{x})(v)$ implying the existence of $\left(t_{k}\right) \downarrow 0$ and $\left(v_{k}, u_{k}\right) \rightarrow(v, u)$ with $\left(\bar{y}+t_{k} v_{k}, \bar{x}+t_{k} u_{k}\right) \in \operatorname{gph} M$ for all $k \in \mathbb{N}$. Particularly, this means $\bar{y}+t_{k} v_{k} \in \operatorname{dom} M$ for all $k \in \mathbb{N}$ and, thus, $v \in$ $T_{\text {dom } M}(\bar{y})$.

On the other hand, consider $v \in T_{\operatorname{dom} M}(\bar{y})$. If $M$ is inner calm* at $\bar{y}$ w.r.t. $\operatorname{dom} M$ in direction $v$ in the fuzzy sense with modulus $\bar{\kappa}_{v}$, then we find $\left(t_{k}\right) \downarrow 0$ and $\left(v_{k}\right) \rightarrow v$ with $\bar{y}+t_{k} v_{k} \in \operatorname{dom} M$ together with $x_{k} \in M\left(\bar{y}+t_{k} v_{k}\right)$ for all $k \in \mathbb{N}$ and $\bar{x} \in \mathbb{R}^{n}$ such that

$$
\left\|x_{k}-\bar{x}\right\| \leq \kappa t_{k}\left\|v_{k}\right\|
$$

holds for each $\kappa>\bar{\kappa}_{v}$ and all $k \in \mathbb{N}$. Introducing $u_{k}:=\left(x_{k}-\bar{x}\right) / t_{k}$, this means that $\left(\bar{y}+t_{k} v_{k}, \bar{x}+t_{k} u_{k}\right) \in \operatorname{gph} M$ is valid for all $k \in \mathbb{N}$. Additionally, we have $\left\|u_{k}\right\| \leq \kappa\left\|v_{k}\right\|$ for all $k \in \mathbb{N}$, and the boundedness of $\left(v_{k}\right)$ yields the boundedness of $\left(u_{k}\right)$. Thus, we find $u \in \mathbb{R}^{n}$ such that $u \in D M(\bar{y}, \bar{x})(v)$ and $\|u\| \leq \kappa\|v\|$ hold, taking also into account that gph $M$ is locally closed around each $(\bar{y}, \bar{x})$ yielding $(\bar{y}, \bar{x}) \in \operatorname{gph} M$ and, thus, $\bar{x} \in M(\bar{y})$. Hence, $\inf _{\bar{x} \in M(\bar{y})} \inf _{u \in D M(\bar{y}, \bar{x})(v)}\|u\| \leq \kappa\|v\|$ follows, and since $\kappa>\bar{\kappa}_{v}$ was arbitrary, the infimum is also bounded from above by $\bar{\kappa}_{v}\|v\|$. The statement about inner calmness* follows immediately from $\bar{\kappa}_{v} \leq \bar{\kappa}$ for every direction $v$.

Next, let us prove the estimate $\widehat{\mathbf{N}}$. Keeping the statement $\mathbf{T}$ for tangents in mind, it is sufficient to prove that

$$
\left(\bigcup_{\bar{x} \in M(\bar{y})} \operatorname{dom} D M(\bar{y}, \bar{x})\right)^{\circ}=\bigcap_{\bar{x} \in M(\bar{y})} \widehat{D}^{*} M(\bar{y}, \bar{x})(0)
$$

holds in order to show the estimates for regular normals. Exploiting the calculus rules of polarization, this holds whenever $(\operatorname{dom} D M(\bar{y}, \bar{x}))^{\circ}=\widehat{D}^{*} M(\bar{y}, \bar{x})(0)$ is valid for all $\bar{x} \in$ $M(\bar{y})$. Thus, let us fix $\bar{x} \in M(\bar{y})$.

Pick $y^{*} \in(\operatorname{dom} D M(\bar{y}, \bar{x}))^{\circ}$ and consider an arbitrary pair $(v, u) \in T_{\operatorname{gph} M}(\bar{y}, \bar{x})$. Then we particularly have $v \in \operatorname{dom} D M(\bar{y}, \bar{x})$, which yields $\left\langle\left(y^{*}, 0\right),(v, u)\right\rangle=\left\langle y^{*}, v\right\rangle \leq 0$, showing $y^{*} \in \widehat{D}^{*} M(\bar{y}, \bar{x})(0)$. Conversely, fix $\tilde{y}^{*} \in \widehat{D}^{*} M(\bar{y}, \bar{x})(0)$ and consider $\bar{v} \in$ $\operatorname{dom} D M(\bar{y}, \bar{x})$. Then we find $u \in D M(\bar{y}, \bar{x})(v)$, and $\left\langle\tilde{y}^{*}, v\right\rangle=\left\langle\left(\tilde{y}^{*}, 0\right),(v, u)\right\rangle \leq 0$ follows which leads to $\tilde{y}^{*} \in(\operatorname{dom} D M(\bar{y}, \bar{x}))^{\circ}$. 
In order to prove $\mathbf{N}$, consider $y^{*} \in N_{\text {dom } M}(\bar{y})$. By definition, we find sequences $\left(y_{k}\right) \rightarrow$ $\bar{y}$ and $\left(y_{k}^{*}\right) \rightarrow y^{*}$ with $y_{k}^{*} \in \widehat{N}_{\text {dom } M}\left(y_{k}\right)$ for all $k \in \mathbb{N}$. By inner semicompactness of $M$ w.r.t. $\operatorname{dom} M$, there exist $\bar{x} \in \mathbb{R}^{n}$ and a sequence $\left(x_{k}\right) \rightarrow \bar{x}$ with $x_{k} \in M\left(y_{k}\right)$ for all $k \in \mathbb{N}$ (at least along a subsequence without relabelling). The closedness properties of $M$ guarantee $\bar{x} \in M(\bar{y})$. The estimate $\widehat{\mathbf{N}}$ for regular normals yields, in particular, that $y_{k}^{*} \in \widehat{D}^{*} M\left(y_{k}, x_{k}\right)(0)$ holds for all $k \in \mathbb{N}$. Thus, taking the limit yields $y^{*} \in D^{*} M(\bar{y}, \bar{x})(0)$.

Finally, let us prove dN. Therefore, we fix $y^{*} \in N_{\operatorname{dom} M}(\bar{y} ; v)$. By definition, we find sequences $\left(t_{k}\right) \downarrow 0,\left(v_{k}\right) \rightarrow v$, and $\left(y_{k}^{*}\right) \rightarrow y^{*}$ such that $y_{k}^{*} \in \widehat{N}_{\text {dom } M}\left(\bar{y}+t_{k} v_{k}\right)$ holds for all $k \in \mathbb{N}$. Since $M$ is inner semicompact at $\bar{y}$ w.r.t. $\operatorname{dom} M$ in direction $v$, we find a point $\bar{x} \in \mathbb{R}^{n}$ and a sequence $\left(x_{k}\right) \rightarrow \bar{x}$ satisfying $x_{k} \in M\left(\bar{y}+t_{k} v_{k}\right.$ ) for all $k \in \mathbb{N}$ (at least along a subsequence without relabelling). Statement $\widehat{\mathbf{N}}$ yields $y_{k}^{*} \in \widehat{D}^{*} M\left(\bar{y}+t_{k} v_{k}, x_{k}\right)(0)$ for all $k \in \mathbb{N}$. Now, we distinguish two cases.

First, assume that $\left(\left(x_{k}-\bar{x}\right) / t_{k}\right)$ is bounded. In this case, there is some $u \in \mathbb{R}^{n}$ such that $\left(\left(x_{k}-\bar{x}\right) / t_{k}\right) \rightarrow u$ holds along a subsequence (without relabelling). Due to the relation $\left(\bar{y}+t_{k} v_{k}, \bar{x}+t_{k}\left(x_{k}-\bar{x}\right) / t_{k}\right) \in \operatorname{gph} M$ for all $k \in \mathbb{N}$, we find $u \in D M(\bar{y}, \bar{x})(v)$ by taking the limit $k \rightarrow \infty$. Furthermore, due to $y_{k}^{*} \in \widehat{D}^{*} M\left(\bar{y}+t_{k} v_{k}, \bar{x}+t_{k}\left(x_{k}-\bar{x}\right) / t_{k}\right)(0)$ for all $k \in \mathbb{N}$, the definition of the limiting directional coderivative yields $y^{*} \in \widehat{D}^{*} M((\bar{y}, \bar{x}) ;(v, u))(0)$. Note that this argumentation is always possible if $M$ is inner calm* at $\bar{y}$ in direction $v$ w.r.t. $\operatorname{dom} M$ with modulus $\bar{\kappa}_{v}$ since we have $\left\|\left(x_{k}-\bar{x}\right) / t_{k}\right\| \leq \kappa\left\|v_{k}\right\|$ for all $k \in \mathbb{N}$ and each $\kappa>\bar{\kappa}_{v}$ in this case for the sequence $\left(x_{k}\right)$ and the point $\bar{x}$ from Definitions 2.3. Taking the limit $k \rightarrow \infty$ yields $\|u\| \leq \kappa\|v\|$.

Next, we assume that $\left(\left(x_{k}-\bar{x}\right) / t_{k}\right)$ is not bounded. In this case, $t_{k} /\left\|x_{k}-\bar{x}\right\| \rightarrow 0$ needs to hold along a subsequence (without relabelling). Setting $\tilde{u}_{k}:=\left(x_{k}-\bar{x}\right) /\left\|x_{k}-\bar{x}\right\|$ for all $k \in \mathbb{N}$, we find some $\tilde{u} \in \mathcal{S}$ such that $\tilde{u}_{k} \rightarrow \tilde{u}$ holds along a subsequence (without relabelling again). Observing that we have

$$
\left(\bar{y}+\left\|x_{k}-\bar{x}\right\| \frac{t_{k} v_{k}}{\left\|x_{k}-\bar{x}\right\|}, \bar{x}+\left\|x_{k}-\bar{x}\right\| \tilde{u}_{k}\right) \in \operatorname{gph} M
$$

for all $k \in \mathbb{N}, \tilde{u} \in D M(\bar{y}, \bar{x})(0)$ follows by taking the limit $k \rightarrow \infty$. Due to

$$
y_{k}^{*} \in \widehat{D}^{*} M\left(\bar{y}+\left\|x_{k}-\bar{x}\right\| \frac{t_{k} v_{k}}{\left\|x_{k}-\bar{x}\right\|}, \bar{x}+\left\|x_{k}-\bar{x}\right\| \tilde{u}_{k}\right)(0)
$$

for all $k \in \mathbb{N}, y^{*} \in D^{*} M((\bar{y}, \bar{x}) ;(0, \tilde{u}))(0)$ is obtained by taking the limit $k \rightarrow \infty$.

Let us recall again that the local closedness of $\operatorname{dom} M$ is automatically satisfied if $M$ is inner semicompact at $\bar{y}$ w.r.t. $\operatorname{dom} M$.

Note that the above theorem extends the results from [4, Theorem 4.1] and [6, Theorem 3.2].

The formulas in Theorem 3.1 reveal an interesting feature: Tangents to the domain can be expressed as a domain of the graphical derivative. On the other hand, normals to the domain are estimated via the image (of 0 ) of the coderivative. In other words, the primal constructions preserve the domain while the dual ones flip it to the image.

Now, let us deal with the other pattern connecting tangents and normals to images of $M$ and the graph of $M$, respectively, by exploiting calmness.

Theorem 3.2 Assume that $M: \mathbb{R}^{m} \rightrightarrows \mathbb{R}^{n}$ possesses a locally closed graph around $(\bar{y}, \bar{x}) \in$ gph $M$. Then the following assertions hold. 
T Tangents: We always have

$$
T_{M(\bar{y})}(\bar{x}) \subset D M(\bar{y}, \bar{x})(0),
$$

and the opposite inclusion holds true whenever $M$ is calm at $(\bar{y}, \bar{x})$.

$\widehat{\mathbf{N}}$ Regular normals: We always have

$$
\widehat{N}_{M(\bar{y})}(\bar{x}) \supset-\operatorname{dom} \widehat{D}^{*} M(\bar{y}, \bar{x}) .
$$

$\mathbf{N}$ Limiting normals: If $M$ is calm at $(\bar{y}, \bar{x})$ with modulus $\bar{\kappa}>0$, then we have

$$
\begin{aligned}
N_{M(\bar{y})}(\bar{x}) & \subset\left\{-x^{*} \mid \inf _{y^{*} \in D^{*} M(\bar{y}, \bar{x})\left(x^{*}\right)}\left\|y^{*}\right\| \leq \bar{\kappa}\left\|x^{*}\right\|\right\} \\
& \subset-\operatorname{dom} D^{*} M(\bar{y}, \bar{x}) .
\end{aligned}
$$

$\mathbf{d N}$ Directional limiting normals: Let $u \in \mathbb{R}^{n}$ be a fixed direction. If $M$ is calm at $(\bar{y}, \bar{x})$ in direction $u$ with modulus $\bar{\kappa}_{u}>0$, then we have

$$
\begin{aligned}
N_{M(\bar{y})}(\bar{x} ; u) & \subset\left\{-\left.x^{*}\right|_{y^{*} \in D^{*} M((\bar{y}, \bar{x}) ;(0, u))\left(x^{*}\right)}\left\|y^{*}\right\| \leq \bar{\kappa}_{u}\left\|x^{*}\right\|\right\} \\
& \subset-\operatorname{dom} D^{*} M((\bar{y}, \bar{x}) ;(0, u)) .
\end{aligned}
$$

Proof Converting [19, Proposition 4.1] from the setting of metric subregularity into the calmness setting readily yields the desired equality in the statement $\mathbf{T}$ as well as the estimate

$$
N_{M(\bar{y})}(\bar{x} ; u) \subset\left\{-x^{*} \mid \exists y^{*} \in D^{*} M((\bar{y}, \bar{x}) ;(0, u))\left(x^{*}\right):\left\|y^{*}\right\| \leq \kappa\left\|x^{*}\right\|\right\}
$$

for any $\kappa>\bar{\kappa}_{u}$. Thus, in particular, the infimum of $\left\|y^{*}\right\|$ over $y^{*} \in D^{*} M((\bar{y}, \bar{x}) ;(0, u))\left(x^{*}\right)$ is bounded by $\kappa\left\|x^{*}\right\|$ and, consequently, also by $\bar{\kappa}_{u}\left\|x^{*}\right\|$. This justifies the statement $\mathbf{d N}$, and $\mathbf{N}$ follows as a special case by choosing $u=0$, see also [25, Theorem 4.1]. The inclusion in $\widehat{\mathbf{N}}$ is obtained by polarization from the inclusion $\subset$ in $\mathbf{T}$ which is generally valid:

$$
\begin{aligned}
\widehat{N}_{M(\bar{y})}(\bar{x}) & \supset(D M(\bar{y}, \bar{x})(0))^{\circ}=\left\{x^{*} \mid\left\langle x^{*}, u\right\rangle \leq 0 \forall u \in D M(\bar{y}, \bar{x})(0)\right\} \\
= & \left\{x^{*} \mid \exists y^{*} \in \mathbb{R}^{m}:\left\langle\left(y^{*}, x^{*}\right),(0, u)\right\rangle \leq 0 \forall(0, u) \in T_{\operatorname{gph} M}(\bar{y}, \bar{x})\right\} \\
& \supset\left\{x^{*} \mid \exists y^{*} \in \mathbb{R}^{m}:\left(y^{*}, x^{*}\right) \in \widehat{N}_{\operatorname{gph} M}(\bar{y}, \bar{x})\right\} \\
= & -\operatorname{dom} \widehat{D}^{*} M(\bar{y}, \bar{x}) .
\end{aligned}
$$

This already completes the proof.

Note that the local closedness of $M(\bar{y})$ around $\bar{x}$ follows from the local closedness of gph $M$ around $(\bar{y}, \bar{x})$.

We see similar features in play as before, namely that tangents to image sets are given via an image of the graphical derivative (the primal constructions preserve the image) while normals to image sets are characterized via the domain of the associated coderivative (the dual constructions flip the image to the domain).

Remark 3.3 Let us mention that Theorems 3.1 and 3.2 are in fact special cases of the calculus rules for tangents and normals to image sets and pre-image sets, respectively, since $\operatorname{dom} M=\varphi(\operatorname{gph} M)$ where $\varphi: \mathbb{R}^{m} \times \mathbb{R}^{n} \rightarrow \mathbb{R}^{m}$ is given by $\varphi(y, x):=y$, while $M(\bar{y})=$ $\phi^{-1}(\operatorname{gph} M)$ for $\phi: \mathbb{R}^{n} \rightarrow \mathbb{R}^{m} \times \mathbb{R}^{n}$ given by $\phi(x):=(\bar{y}, x)$. Related results for tangents 
and (directional) limiting normals can be found in [4, Corollary 4.2 and Theorem 4.3] and [6, Theorems 3.1 and 3.2], where the inner calmness* assumption is imposed on

$$
\tilde{M}_{1}(y):=\varphi^{-1}(y) \cap \operatorname{gph} M=(y, M(y))
$$

and the calmness assumption is imposed on

$$
\tilde{M}_{2}(y, z):=\{x \mid \phi(x)+(y, z) \in \operatorname{gph} M\}=M(\bar{y}+y)-z .
$$

Since the functions $y \rightarrow y,(y, z) \rightarrow \bar{y}+y$, and $(y, z) \rightarrow-z$ are affine, they are calm, and Proposition 2.6 yields that the inner calmness* of $M$ implies the inner calmness* of $\widetilde{M}_{1}$ and the calmness of $M$ implies that of $\widetilde{M}_{2}$. In fact, these assumptions are equivalent; the inner calmness* of $M$ follows immediately from the inner calmness* of $\widetilde{M}_{1}$, while the calmness of $M$ follows again from Proposition 2.6 since $M(y)=\widetilde{M}_{2}(y-\bar{y}, 0)$.

Finally, let us comment on some potential extensions of our results to the setting of Asplund spaces where the calculus of (directional) limiting normals is well-developed as well, see $[36,40]$.

Remark 3.4 Let us assume that the set-valued mapping under consideration acts between Asplund spaces which are Banach spaces where all continuous, convex functions are generically Fréchet differentiable, see [40] for details.

For simplicity, we start with a review of Theorem 3.2. One can easily check that statement $\mathbf{T}$ holds as stated in the more general situation. This, however, cannot be used to prove a counterpart of $\widehat{\mathbf{N}}$ since the regular normal cone is only a subset of the polar associated with the tangent cone in general. In reflexive spaces, we get full polarity only w.r.t. the weak tangent cone, where weak convergence of the directions is demanded in the definition, see [40, Theorem 1.10, Corollary 1.11]. In order to transfer $\mathbf{N}$ and $\mathbf{d N}$ to the Asplund space setting, the underlying proof from [19] has to be adjusted slightly in order to handle that (directional) limiting normals are weak* limits of regular normals in the infinite-dimensional situation, see [36, Section 2] and [40, Section 3] for details.

Let us now commend on Theorem 3.1. Here, the inclusion $\supset$ in statement $\mathbf{T}$ remains valid in the more general setting. The converse inclusions, however, cannot be shown in the presented way since bounded sequences in infinite-dimensional spaces do not possess convergent subsequences in general. Even in the setting of reflexive Banach spaces, this issue cannot be solved since one constructs elements of the possibly larger weak tangent cone in the proof. A similar reasoning can be used to infer that the proof of statement $\mathbf{d N}$ does not apply in infinite dimensions. It is not clear how to obtain any of the inclusions of statement $\widehat{\mathbf{N}}$ due to the already mentioned difficulties. On the other hand, one can easily check that statement $\mathbf{N}$ stays true when using the mixed coderivative of the involved set-valued map, see [40, Definition 1.32] for a definition.

To sum up, a full generalization of the patterns observed in Theorems 3.1 and 3.2 to the infinite-dimensional situation is highly questionable for regular normals. It might be possible to carry over parts of the analysis for regular normals to so-called Dini-Hadamard normals which are, by definition, polar to tangents, see [43] for details. The situation seems to be less hopeless for tangents and directional limiting normals in reflexive spaces. However, one has to find a way to bypass the appearance of weak tangents. For limiting normals, an extension to Asplund spaces seems to be possible, directly. Nevertheless, we would like to point the reader's attention to the fact that due to certain convexification effects, limiting normals turned out to be of limited practical use in Lebesgue and Sobolev spaces which are standard in optimal control, see $[24,37,38]$ for a detailed investigation. 


\section{Sufficient Conditions for Calmness and Inner Calmness*}

In this section, we connect calmness and inner calmness* with several other continuity and Lipschitzian properties of set-valued mappings. We begin, however, by showing that inner calmness* in the fuzzy sense is not only a sufficient condition for validity of the characterization of tangents to the domain of a set-valued mapping from Theorem 3.1, but that the two are actually equivalent.

Theorem 4.1 Let $M: \mathbb{R}^{m} \rightrightarrows \mathbb{R}^{n}$ be a set-valued mapping and fix $\bar{y} \in \operatorname{dom} M$ such that gph $M$ is locally closed around $\{\bar{y}\} \times \mathbb{R}^{n}$ and $\operatorname{dom} M$ is locally closed around $\bar{y}$. Then $M$ is inner calm* at $\bar{y}$ in direction $v \in \mathcal{S}$ w.r.t. $\operatorname{dom} M$ with modulus $\bar{\kappa}_{v}$ in the fuzzy sense if and only if

$$
v \in T_{\operatorname{dom} M}(\bar{y}) \Longrightarrow \bar{\kappa}_{v}=\inf _{\bar{x} \in M(\bar{y})} \inf _{u \in D M(\bar{y}, \bar{x})(v)}\|u\| /\|v\|<\infty .
$$

In particular, $M$ is inner calm* at $\bar{y}$ w.r.t. dom $M$ in the fuzzy sense if and only if (4.1) holds for every direction $v \in \mathcal{S}$.

Proof For the proof, we set

$$
\hat{\kappa}_{v}:=\inf _{\bar{x} \in M(\bar{y})} \inf _{u \in D M(\bar{y}, \bar{x})(v)}\|u\| /\|v\| .
$$

First, assume that $M$ is inner calm* at $\bar{y}$ in direction $v$ w.r.t. dom $M$ in the fuzzy sense with modulus $\bar{\kappa}_{v}$. Then Theorem 3.1 yields the finiteness of $\hat{\kappa}_{v}$ as well as $\hat{\kappa}_{v} \leq \bar{\kappa}_{v}$. Supposing that $\hat{\kappa}_{v}<\bar{\kappa}_{v}$ holds, we find some $\varepsilon>0, \bar{x} \in M(\bar{y})$, and $u \in \mathbb{R}^{n}$ as well as sequences $\left(t_{k}\right) \downarrow 0$, $\left(u_{k}\right) \rightarrow u$, and $\left(v_{k}\right) \rightarrow v$ such that $\left(\bar{y}+t_{k} v_{k}, \bar{x}+t_{k} u_{k}\right) \in \operatorname{gph} M$ and $\left\|u_{k}\right\| \leq\left(\bar{\kappa}_{v}-\varepsilon\right)\left\|v_{k}\right\|$ hold for all $k \in \mathbb{N}$. Setting $y_{k}:=\bar{y}+t_{k} v_{k}$ and $x_{k}:=\bar{x}+t_{k} u_{k}$ for all $k \in \mathbb{N}$, we find $\left(y_{k}\right) \rightarrow \bar{y},\left(x_{k}\right) \rightarrow \bar{x}$, and $y_{k} \in \operatorname{dom} M$ as well as $\left\|x_{k}-\bar{x}\right\| \leq\left(\bar{\kappa}_{v}-\varepsilon\right)\left\|y_{k}-\bar{y}\right\|$ for all $k \in \mathbb{N}$. This contradicts the definition of the modulus of inner calmness* of $M$ at $\bar{y}$ w.r.t. $\operatorname{dom} M$ in direction $v$ in the fuzzy sense. Hence, we have shown the validity of (4.1).

Next, assume that (4.1) holds. If $v \notin T_{\operatorname{dom} M}(\bar{y})$ is valid, there is nothing to prove. Thus, let us assume $v \in T_{\operatorname{dom} M}(\bar{y})$. Then (4.1) yields the existence of $\bar{x} \in M(\bar{y})$ and $u \in \mathbb{R}^{n}$ together with sequences $\left(t_{k}\right) \downarrow 0,\left(u_{k}\right) \rightarrow u$, and $\left(v_{k}\right) \rightarrow v$ such that $\left(\bar{y}+t_{k} v_{k}, \bar{x}+t_{k} u_{k}\right) \in$ gph $M$ and $\left\|u_{k}\right\| \leq \kappa\left\|v_{k}\right\|$ hold for all $\kappa>\hat{\kappa}_{v}$ and sufficiently large $k \in \mathbb{N}$. We set $y_{k}:=$ $\bar{y}+t_{k} v_{k}$ and $x_{k}:=\bar{x}+t_{k} u_{k}$ for all $k \in \mathbb{N}$. Hence, $\left\|x_{k}-\bar{x}\right\| \leq \kappa\left\|y_{k}-\bar{y}\right\|$ is valid for all $\kappa>\hat{\kappa}_{v}$ and sufficiently large $k \in \mathbb{N}$ showing the inner calmness* of $M$ at $\bar{y}$ in the fuzzy sense w.r.t. dom $M$ in direction $v$ while the modulus satisfies $\bar{\kappa}_{v} \leq \hat{\kappa}_{v}$. The first part of the proof now yields $\bar{\kappa}_{v}=\hat{\kappa}_{v}$.

Below, we recall some prominent continuity and Lipschitzianity notions from the literature, see e.g. [6, 14, 40].

Definition 4.2 Consider a set-valued mapping $M: \mathbb{R}^{m} \rightrightarrows \mathbb{R}^{n}$ which possesses a locally closed graph around $(\bar{y}, \bar{x}) \in \operatorname{gph} M$. We say that

(i) $\quad M$ is inner semicontinuous at $(\bar{y}, \bar{x})$ w.r.t. a set $\Omega \subset \mathbb{R}^{m}$ if for every sequence $\left(y_{k}\right) \subset$ $\Omega$ satisfying $\left(y_{k}\right) \rightarrow \bar{y}$, there exists a sequence $\left(x_{k}\right) \rightarrow \bar{x}$ such that $x_{k} \in M\left(y_{k}\right)$ holds for sufficiently large $k \in \mathbb{N}$. In case $\Omega:=\mathbb{R}^{m}$, we simply say that $M$ is inner semicontinuous at $(\bar{y}, \bar{x})$. 
(ii) $\quad M$ is inner calm at $(\bar{y}, \bar{x})$ w.r.t. a set $\Omega \subset \mathbb{R}^{m}$ if there exists $\kappa>0$ such that for every sequence $\left(y_{k}\right) \subset \Omega$ satisfying $\left(y_{k}\right) \rightarrow \bar{y}$, there exists a sequence $\left(x_{k}\right) \rightarrow \bar{x}$ such that $x_{k} \in M\left(y_{k}\right)$ and

$$
\left\|x_{k}-\bar{x}\right\| \leq \kappa\left\|y_{k}-\bar{y}\right\|
$$

hold for sufficiently large $k \in \mathbb{N}$. In case $\Omega:=\mathbb{R}^{m}$, we simply say that $M$ is inner calm at $(\bar{y}, \bar{x})$.

(iii) $\quad M$ has the isolated calmness property at $(\bar{y}, \bar{x})$ if there exist $\kappa>0$ and neighborhoods $U$ of $\bar{x}$ and $V$ of $\bar{y}$ such that the following estimate holds:

$$
M(y) \cap U \subset \bar{x}+\kappa\|y-\bar{y}\| \mathcal{B} \quad \forall y \in V .
$$

(iv) $\quad M$ has the Aubin property at $(\bar{y}, \bar{x})$ if there exist $\kappa>0$ and neighborhoods $U$ of $\bar{x}$ and $V$ of $\bar{y}$ such that the following estimate holds:

$$
M\left(y^{\prime}\right) \cap U \subset M(y)+\kappa\left\|y-y^{\prime}\right\| \mathcal{B} \quad \forall y, y^{\prime} \in V .
$$

The modulus of inner calmness (w.r.t. $\Omega$ ), isolated calmness, and the Aubin property, respectively, is defined as the infimum over all $\kappa$ satisfying the respective Lipschitz estimate from above.

Let $M: \mathbb{R}^{m} \rightrightarrows \mathbb{R}^{n}$ be a set-valued mapping with locally closed graph around some point $(\bar{y}, \bar{x}) \in$ gph $M$. Inner semicontinuity and inner calmness at $(\bar{y}, \bar{x})$ are clearly stronger than inner semicompactness and inner calmness* at $\bar{y}$, respectively. Thus, in Theorem 3.1, we focused on inner semicompactness, and inner calmness* (in the fuzzy sense) in order to have the results more general, taking into account that it is very easy to derive the estimates based on inner semicontinuity and inner calmness - one just has the fixed $\bar{x}$ instead of the unions. More importantly, it seems that these weaker notions have a better chance to be satisfied in practically relevant settings, see [4, Section 3].

Let us note that $M$ is inner calm at $(\bar{y}, \bar{x}) \in \operatorname{gph} M$ if and only if there are a constant $\kappa>0$ as well as a neighborhood $V$ of $\bar{y}$ such that

$$
\bar{x} \in M(y)+\kappa\|y-\bar{y}\| \mathcal{B} \quad \forall y \in V
$$

holds. Thus, whenever $M$ possesses the Aubin property at $(\bar{y}, \bar{x})$, it is also inner calm there. However, we note that the modulus of inner calmness might be strictly smaller than the modulus of the Aubin property in this situation. It is both obvious and well known that whenever $M$ is isolatedly calm at $(\bar{y}, \bar{x})$ or possesses the Aubin property at this point, then $M$ is also calm there. More precisely, isolated calmness implies calmness with the same modulus, while the Aubin property implies calmness with not larger modulus.

Observe that (isolated) calmness and inner calmness of a set-valued mapping are not related to each other. On the one hand, the single-valued function $m: \mathbb{R} \rightarrow \mathbb{R}$, given by $m(y):=1 / y$ if $y \in \mathbb{R} \backslash\{0\}$ and $m(0):=0$, is isolatedly calm at $(0,0) \in \operatorname{gph} m$, and hence also calm, but it is not inner calm there. On the other hand, $M: \mathbb{R} \rightrightarrows \mathbb{R}$ given by $M(y):=\left[-|y|^{1 / 2}, \infty\right)$ for all $y \in \mathbb{R}$ is inner calm but not calm at $(0,0)$, let alone isolatedly calm. The following lemma, however, shows that under additional assumptions, isolated calmness may imply inner calmness or inner calmness* (in the fuzzy sense).

Lemma 4.3 Let $M: \mathbb{R}^{m} \rightrightarrows \mathbb{R}^{n}$ be a set-valued mapping whose graph is locally closed around $\{\bar{y}\} \times \mathbb{R}^{n}$ for some $\bar{y} \in \operatorname{dom} M$. Then the following assertions hold.

(i) Fix a point $\bar{x} \in M(\bar{y})$. If $M$ is inner semicontinuous w.r.t. $\operatorname{dom} M$ and isolatedly calm at $(\bar{y}, \bar{x})$, then it is inner calm w.r.t. $\operatorname{dom} M$ there. 
(ii) If $M$ is inner semicompact at $\bar{y}$ w.r.t. $\operatorname{dom} M$ and isolatedly calm at $(\bar{y}, x)$ for each $x \in M(\bar{y})$, then it is inner calm* at $\bar{y}$ w.r.t. $\operatorname{dom} M$.

Proof Let us start with the proof of (i). Since $M$ is isolatedly calm at $(\bar{y}, \bar{x})$, we find a constant $\kappa>0$ and neighborhoods $U$ of $\bar{x}$ as well as $V$ of $\bar{y}$ satisfying (4.2). Given $\left(y_{k}\right) \subset$ $\operatorname{dom} M$ with $\left(y_{k}\right) \rightarrow \bar{y}$, we find a sequence $\left(x_{k}\right) \rightarrow \bar{x}$ satisfying $x_{k} \in M\left(y_{k}\right)$ for all $k \in \mathbb{N}$ by inner semicontinuity of $M$ at $(\bar{y}, \bar{x})$ w.r.t. $\operatorname{dom} M$. Due to (4.2), we find $\left\|x_{k}-\bar{x}\right\| \leq \kappa\left\|y_{k}-\bar{x}\right\|$ for large enough $k \in \mathbb{N}$ showing the inner calmness of $M$ at $(\bar{y}, \bar{x})$ w.r.t. $\operatorname{dom} M$.

Next, we show (ii). First, since points $x \in M(\bar{y})$ are isolated, there can be only countably many of them, i.e., $M(\bar{y})=\left\{x^{l} \mid l \in N\right\}$ for some $N \subset \mathbb{N}$. For each $l \in N$ let $\varepsilon_{l}>0$ be such that $M(\bar{y}) \cap\left(x^{l}+\varepsilon_{l} \mathcal{B}\right)=\left\{x^{l}\right\}$. By inner semicompactness of $M$ at $\bar{y}$ w.r.t. $\operatorname{dom} M$, for every $\left(y_{k}\right) \subset \operatorname{dom} M$ with $\left(y_{k}\right) \rightarrow \bar{y}$, we find a sequence $\left(x_{k}\right)$ which converges along a subsequence to some $\tilde{x} \in \mathbb{R}^{n}$ such that $x_{k} \in M\left(y_{k}\right)$ holds along this subsequence. By closedness of gph $M$ around $\{\bar{y}\} \times \mathbb{R}^{n}$, we find $\tilde{x}=x^{l}$ for some $l \in N$, and we denote by $l\left(y_{k}\right)$ the smallest of these numbers, i.e.,

$$
l\left(y_{k}\right):=\min \left\{l \in N \mid \liminf _{k \rightarrow \infty} \operatorname{dist}\left(x^{l}, M\left(y_{k}\right)\right)=0\right\} .
$$

In particular, for each $j \in N$ with $j<l\left(y_{k}\right)$, we get $\liminf _{k \rightarrow \infty} \operatorname{dist}\left(x^{j}, M\left(y_{k}\right)\right) \geq \varepsilon_{j} / 2>$ 0 . Let us now prove that the set

$$
\tilde{N}:=\left\{l \in N \mid \exists\left(y_{k}^{l}\right) \subset \operatorname{dom} M:\left(y_{k}^{l}\right) \rightarrow \bar{y}, l=l\left(y_{k}^{l}\right)\right\}
$$

is finite. By contraposition, suppose that $\widetilde{N}$ is infinite and for each $l \in \widetilde{N}$, let $k_{l}$ be such that $\left\|y_{k_{l}}^{l}-\bar{y}\right\| \leq 1 / l$ and $\operatorname{dist}\left(x^{j}, M\left(y_{k_{l}}^{l}\right)\right) \geq \varepsilon_{j} / 4>0$ for all $j \in N$ with $j<l$. Consider the sequence $\left(y_{k_{l}}^{l}\right) \subset \operatorname{dom} M$ for indices $l \in \tilde{N}$. By construction, $\left(y_{k_{l}}^{l}\right) \rightarrow \bar{y}$ holds as $l \rightarrow \infty$. Then, for each $j \in N$, we have $\operatorname{dist}\left(x^{j}, M\left(y_{k_{l}}^{l}\right)\right) \geq \varepsilon_{j} / 4>0$ for all $l \in \tilde{N}$ with $l>j$ and, consequently, $\liminf \operatorname{in}_{l \rightarrow \infty} \operatorname{dist}\left(x^{j}, M\left(y_{k_{l}}^{l}\right)\right) \geq \varepsilon_{j} / 4>0$ follows. This, however, contradicts the assumed inner semicompactness of $M$.

The rest of the proof now follows easily. Since $M$ is isolatedly calm at $\left(\bar{y}, x^{l}\right)$ for each $l \in \tilde{N}$, we find constants $\kappa_{l}>0$ and neighborhoods $U_{l}$ of $x^{l}$ as well as $V_{l}$ of $\bar{y}$ such that (4.2) holds with $\kappa:=\max _{l \in \widetilde{N}} \kappa_{l}, U:=U_{l}$, and $V:=V_{l}$ for each $l \in \widetilde{N}$. Consider a sequence $\left(y_{k}\right) \subset \operatorname{dom} M$ with $\left(y_{k}\right) \rightarrow \bar{y}$ together with the corresponding point $x^{l\left(y_{k}\right)} \in M(\bar{y})$ and sequence $\left(x_{k}\right)$ such that $x_{k} \in M\left(y_{k}\right)$ and $\left(x_{k}\right) \rightarrow x^{l\left(y_{k}\right)}$ hold along a subsequence. Since $l\left(y_{k}\right) \in \widetilde{N}$ holds, we obtain $\left\|x_{k}-x^{l\left(y_{k}\right)}\right\| \leq \kappa\left\|y_{k}-\bar{y}\right\|$, showing that $M$ is inner calm* at $\bar{y}$ w.r.t. $\operatorname{dom} M$.

We would like to point out again that the actual modulus of inner calmness (inner calmness*) can be smaller than the (supremum of the) underlying modulus (moduli) of isolated calmness. Exemplary, by means of the set-valued mapping $M: \mathbb{R} \rightrightarrows \mathbb{R}$ given by $M(y):=\{k(y+1) \mid k \in \mathbb{N}\}$ for each $y \in \mathbb{R}$, one can easily check that the assumptions of setting (ii) hold at $\bar{y}:=0$. Particularly, $M$ is isolatedly calm at each point $(0, k), k \in \mathbb{N}$, with modulus $k$. The supremum of all these moduli is, obviously, not finite. On the other hand, $M$ is inner calm* at $\bar{y}$ with modulus 1 .

The main reason why we consider isolated calmness and the Aubin property is that these conditions can be characterized via generalized derivatives as follows. For a set-valued mapping $M: \mathbb{R}^{m} \rightrightarrows \mathbb{R}^{n}$ possessing locally closed graph around $(\bar{y}, \bar{x}) \in \operatorname{gph} M$, isolated calmness of $M$ at $(\bar{y}, \bar{x})$ is equivalent to

$$
\operatorname{DM}(\bar{y}, \bar{x})(0)=\{0\},
$$


see [35], which is referred to as Levy-Rockafellar criterion in the literature. On the other hand, $M$ possesses the Aubin property at $(\bar{y}, \bar{x})$ if and only if

$$
D^{*} M(\bar{y}, \bar{x})(0)=\{0\}
$$

is valid, see [46, Theorem 9.40], and the latter is known as Mordukhovich criterion. We note that the Aubin property can be characterized in terms of the graphical derivative as well, see [2, Theorem 7.5.4] and [14, Theorem 4B.2].

Consider a single-valued continuous function $\varphi: \mathbb{R}^{n} \rightarrow \mathbb{R}^{m}$. For arbitrary $\bar{x} \in \mathbb{R}^{n}$, it is well known that the Aubin property of $\varphi$ at $(\bar{x}, \varphi(\bar{x}))$ equals local Lipschitzness of $\varphi$ at $\bar{x}$ which is, thus, characterized by $D^{*} \varphi(\bar{x})(0)=\{0\}$. On the other hand, since $\varphi$ is inner semicontinuous at each point of its graph, calmness, inner calmness, and isolated calmness coincide and correspond to the standard definition of calmness of single-valued mappings, see Section 2.2. Consequently, we obtain the following simple corollary, see also [46, Proposition 9.24].

Corollary 4.4 Fix $\bar{x} \in \mathbb{R}^{n}$. A continuous function $\varphi: \mathbb{R}^{n} \rightarrow \mathbb{R}^{m}$ is calm at $\bar{x}$ if and only if $D \varphi(\bar{x})(0)=0$ holds.

Exemplary, consider the continuous function $\varphi: \mathbb{R} \rightarrow \mathbb{R}$ given by $\varphi(x):=x^{3 / 2} \sin (1 / x)$ for all $x \in \mathbb{R} \backslash\{0\}$ and $\varphi(0):=0$. We find $D^{*} \varphi(0)(0)=\mathbb{R}$ and $D \varphi(0)(0)=\{0\}$, i.e., $\varphi$ is calm at 0 but not Lipschitz at 0 .

Let us now review Theorem 3.1 in the light of isolated calmness and the Aubin property. Lemma 4.3 yields the following result in terms of isolated calmness.

Corollary 4.5 Assume that $M: \mathbb{R}^{m} \rightrightarrows \mathbb{R}^{n}$ has locally closed graph around $\{\bar{y}\} \times \mathbb{R}^{n}$ for some $\bar{y} \in \operatorname{dom} M$, that $M$ is inner semicompact at $\bar{y}$ w.r.t. $\operatorname{dom} M$, and that (LRC) holds for each $\bar{x} \in M(\bar{y})$. Let $v \in \mathbb{R}^{m}$ be an arbitrary direction. Then we have the relations

$$
\begin{aligned}
T_{\operatorname{dom} M}(\bar{y}) & =\bigcup_{\bar{x} \in M(\bar{y})} \operatorname{dom} D M(\bar{y}, \bar{x}), \\
\widehat{N}_{\operatorname{dom} M}(\bar{y}) & =\bigcap_{\bar{x} \in M(\bar{y})} \widehat{D}^{*} M(\bar{y}, \bar{x})(0), \\
N_{\operatorname{dom} M}(\bar{y}) & \subset \bigcup_{\bar{x} \in M(\bar{y})} D^{*} M(\bar{y}, \bar{x})(0), \\
N_{\operatorname{dom} M}(\bar{y} ; v) & \subset \bigcup_{\bar{x} \in M(\bar{y})} \bigcup_{u \in D M(\bar{y}, \bar{x})(v)} D^{*} M((\bar{y}, \bar{x}) ;(v, u))(0) .
\end{aligned}
$$

Let us now revisit Theorem 3.1 in terms of the Aubin property whose presence is also sufficient for validity of inner calmness. Clearly, if $M: \mathbb{R}^{m} \rightrightarrows \mathbb{R}^{n}$ possesses the Aubin property at some point $(\bar{y}, \bar{x}) \in \operatorname{gph} M$ where gph $M$ is locally closed, then, on the one hand, we find $\bar{y} \in$ int $\operatorname{dom} M$, i.e., $T_{\operatorname{dom} M}(\bar{y})=\mathbb{R}^{m}$ and $\widehat{N}_{\operatorname{dom} M}(\bar{y})=N_{\operatorname{dom} M}(\bar{y})=$ $N_{\text {dom } M}(\bar{y} ; v)=\{0\}$ hold for all $v \in \mathbb{R}^{m}$. On the other hand, Theorem 3.1 reproduces these trivial relations for limiting normals and directional limiting normals, taking into account that the estimates hold without the union (i.e., with the fixed $\bar{x}$ ). Observing that the regular coderivative is a subset of the limiting one, we also recover $\widehat{N}_{\operatorname{dom} M}(\bar{y})=\{0\}$. Finally, from $N_{\text {dom } M}(\bar{y})=\{0\}$, we find $\mathbb{R}^{m}=\left(N_{\operatorname{dom} M}(\bar{y})\right)^{\circ} \subset T_{\operatorname{dom} M}(\bar{y})$ where the last inclusion follows from [46, Theorem 6.26, Exercise 6.38]. 
The situation is quite similar if we look at Theorem 3.2 assuming that $M$ is isolatedly calm at $(\bar{y}, \bar{x})$. In this case, (4.2) guarantees that $\bar{x}$ is an isolated point of $M(\bar{y})$ which shows $T_{M(\bar{y})}(\bar{x})=\{0\}$ and $\widehat{N}_{M(\bar{y})}(\bar{x})=N_{M(\bar{y})}(\bar{x})=N_{M(\bar{y})}(\bar{x} ; 0)=\mathbb{R}^{n}$. Using the LevyRockafellar criterion (LRC), Theorem 3.2 recovers this observation for tangents. This yields $\widehat{N}_{M(\bar{y})}(\bar{x})=\mathbb{R}^{n}$ and, thus, $N_{M(\bar{y})}(\bar{x})=N_{M(\bar{y})}(\bar{x} ; 0)=\mathbb{R}^{n}$ (note that 0 is the only vector in $\left.T_{M(\bar{y})}(\bar{x})\right)$. Thus, Theorem 3.2 implies $\operatorname{dom} D^{*} M(\bar{y}, \bar{x})=\operatorname{dom} D^{*} M((\bar{y}, \bar{x}) ;(0,0))=\mathbb{R}^{n}$. Observe that we do not obtain any information about the domain of the regular coderivative $\widehat{D}^{*} M(\bar{y}, \bar{x})$. Exemplary, take a look at the mappings $M_{1}, M_{2}: \mathbb{R} \rightrightarrows \mathbb{R}$ given by $M_{1}(y):=$ $[-|y|,|y|]$ and $M_{2}(y):=\left[-y^{2}, y^{2}\right]$ for all $y \in \mathbb{R}$. Clearly, we have $D M_{i}(0,0)(0)=\{0\}$ for $i=1,2$, i.e., (LRC) holds. On the other hand, we have dom $\widehat{D}^{*} M_{1}(0,0)=\{0\}$ and $\operatorname{dom} \widehat{D}^{*} M_{2}(0,0)=\mathbb{R}$. Thus, (LRC) does not yield a sharp estimate for regular normals. Another way to look at this situation is that due to the fact that isolated calmness of $M$ at $(\bar{y}, \bar{x})$ yields the isolatedness of $\bar{x}$ in $M(\bar{y})$ and, thus, $T_{M(\bar{y})}(\bar{x})=\{0\}$, Theorem 3.2 shows that (LRC) is indeed necessary for isolated calmness.

Using the Aubin property in Theorem 3.2 yields the following estimates.

Corollary 4.6 Assume that $M: \mathbb{R}^{m} \rightrightarrows \mathbb{R}^{n}$ possesses a locally closed graph around $(\bar{y}, \bar{x}) \in$ gph $M$ and that (MC) is valid. Let $u \in \mathbb{R}^{n}$ be an arbitrary direction. Then the following relations hold:

$$
\begin{aligned}
T_{M(\bar{y})}(\bar{x}) & =D M(\bar{y}, \bar{x})(0), \\
\widehat{N}_{M(\bar{y})}(\bar{x}) & \supset-\operatorname{dom} \widehat{D}^{*} M(\bar{y}, \bar{x}), \\
N_{M(\bar{y})}(\bar{x}) & \subset-\operatorname{dom} D^{*} M(\bar{y}, \bar{x}), \\
N_{M(\bar{y})}(\bar{x} ; u) & \subset-\operatorname{dom} D^{*} M((\bar{y}, \bar{x}) ;(0, u)) .
\end{aligned}
$$

Finally, we consider the first-order sufficient condition for calmness (FOSCclm for short) given by

$$
D^{*} M((\bar{y}, \bar{x}) ;(0, u))(0)=\{0\} \quad \forall u \in D M(\bar{y}, \bar{x})(0) \backslash\{0\} .
$$

(FOSCclm)

As mentioned in Section 1, this condition is nothing else but the calmness counterpart of Gfrerer's FOSCMS. The following lemma and its proof justify this fact.

Lemma 4.7 Let $M: \mathbb{R}^{m} \rightrightarrows \mathbb{R}^{n}$ be a set-valued mapping with locally closed graph around $(\bar{y}, \bar{x}) \in \operatorname{gph}$ M. Furthermore, let (FOSCclm) be valid at $(\bar{y}, \bar{x})$. Then $M$ is calm at $(\bar{y}, \bar{x})$.

Proof Recall that $M$ is calm at $(\bar{y}, \bar{x})$ if and only if the inverse mapping $M^{-1}$ is metrically subregular at $(\bar{x}, \bar{y})$. Furthermore, due to [19, Section 2.2], the condition

$$
0 \in D^{*} M^{-1}((\bar{x}, \bar{y}) ;(u, 0))\left(y^{*}\right) \Longrightarrow y^{*}=0 \quad \forall u \neq 0: \quad 0 \in D M^{-1}(\bar{x}, \bar{y})(u)
$$

is sufficient for metric subregularity of $M^{-1}$ at $(\bar{x}, \bar{y})$. By the definition of the inverse mapping and Lemma 2.1, we find $u \in D M(\bar{y}, \bar{x})(0) \Longleftrightarrow 0 \in D M^{-1}(\bar{x}, \bar{y})(u)$ for all $u \in \mathbb{R}^{n}$ and

$$
-y^{*} \in D^{*} M((\bar{y}, \bar{x}) ;(0, u))(0) \Longleftrightarrow 0 \in D^{*} M^{-1}((\bar{x}, \bar{y}) ;(u, 0))\left(y^{*}\right)
$$

for all $y^{*} \in \mathbb{R}^{m}$, showing that (FOSCclm) and (4.3) are equivalent and the proof is done.

Naturally, validity of (FOSCclm) just for some $u$ implies calmness in direction $u$. 
We point out that (FOSCclm) is a refinement of (LRC) and MC, i.e., it is implied by each of them. It turns out that (FOSCclm) also yields all the calculus estimates and yet it is not too strong to make some of them irrelevant.

Theorem 4.8 Let $M: \mathbb{R}^{m} \rightrightarrows \mathbb{R}^{n}$ be a given set-valued mapping and fix $\bar{y} \in \operatorname{dom} M$. Then the following assertions hold.

(i) Assume that $M$ has locally closed graph around $\{\bar{y}\} \times \mathbb{R}^{n}$, that $M$ is inner semicompact at $\bar{y}$ w.r.t. $\operatorname{dom} M$, and that (FOSCclm) holds at $(\bar{y}, \bar{x})$ for each $\bar{x} \in M(\bar{y})$. Then the relations from Corollary 4.5 are valid.

(ii) Fix $\bar{x} \in M(\bar{y})$, let $M$ possess a locally closed graph around $(\bar{y}, \bar{x})$, and assume that (FOSCclm) holds at $(\bar{y}, \bar{x})$. Then the relations from Corollary 4.6 are valid.

Proof Taking Lemma 4.7 into account, assertion (ii) is obvious from Theorem 3.2. Thus, we only need to prove assertion (i).

The estimate for the limiting normals holds true due to the assumed inner semicompactness of $M$ at $\bar{y}$ w.r.t. $\operatorname{dom} M$, see Theorem 3.1. Similarly, inner semicompactness of $M$ at $\bar{y}$ w.r.t. $\operatorname{dom} M$ readily yields the more complicated estimate for the directional limiting normals presented in Theorem 3.1. However, (FOSCclm) guarantees that

$$
\bigcup_{u \in D M(\bar{y}, \bar{x})(0) \cap \mathcal{S}} D^{*} M((\bar{y}, \bar{x}) ;(0, u))(0)
$$

reduces to $\{0\}$ for each $\bar{x} \in M(\bar{y})$ which is why the simpler estimate from Corollary 4.5 holds.

Notably, this actually implies the other two relations for tangents and regular normals. Indeed, given $v \in T_{\operatorname{dom} M}(\bar{y})$, in particular, we have $0 \in N_{\operatorname{dom} M}(\bar{y} ; v) \neq \varnothing$ by definition of the directional limiting normal cone. Hence, the estimate for directional normals yields the existence of $\bar{x} \in M(\bar{y})$ together with $u \in D M(\bar{y}, \bar{x})(v)$, i.e., $v \in \operatorname{dom} D M(\bar{y}, \bar{x})$. Since the opposite inclusion is always valid, see Theorem 3.1, we are done. Finally, the estimate for regular normals follows from polarization, see the proof of Theorem 3.1 as well.

The question remains whether (FOSCclm) only implies the estimates of Corollary 4.5 or also inner calmness* (in the fuzzy sense). Thanks to the equivalence from Theorem 4.1, we can indeed provide a fuzzy version of Lemma 4.3 in terms of (FOSCclm). In order to handle the first statement of Lemma 4.3 as well, we formally introduce the naturally arising concept of inner calmness in the fuzzy sense for the sake of completeness. Expectedly, we say that a set-valued mapping $M: \mathbb{R}^{m} \rightrightarrows \mathbb{R}^{n}$ is inner calm at $(\bar{y}, \bar{x}) \in \operatorname{gph} M$ w.r.t. $\operatorname{dom} M$ in the fuzzy sense if $\operatorname{dom} M$ is locally closed at $\bar{y}$ while for each direction $v \in T_{\operatorname{dom} M}(\bar{y}) \cap \mathcal{S}$, we find a constant $\kappa_{v}>0$, a sequence $\left(y_{k}\right) \subset \operatorname{dom} M$ converging to $\bar{y}$ from $v$, and a sequence $\left(x_{k}\right)$ satisfying $x_{k} \in M(\bar{y})$ as well as $\left\|x_{k}-\bar{x}\right\| \leq \kappa_{v}\left\|y_{k}-\bar{y}\right\|$ for sufficiently large $k \in \mathbb{N}$. This notion follows from the definition of inner calmness* in the fuzzy sense, see Definition 2.4 , by fixing $\bar{x} \in M(\bar{y})$. It is, thus, not surprising that one can state a similar result as in Theorem 4.1 in order to characterize inner calmness in the fuzzy sense with the aid of the graphical derivative of $M$ at $(\bar{y}, \bar{x})$.

Corollary 4.9 Let $M: \mathbb{R}^{m} \rightrightarrows \mathbb{R}^{n}$ be a set-valued mapping and fix $\bar{y} \in \operatorname{dom} M$ such that gph $M$ is locally closed around $\{\bar{y}\} \times \mathbb{R}^{n}$. Then the following assertions hold.

(i) Fix a point $(\bar{y}, \bar{x}) \in \operatorname{gph} M$. If $M$ is inner semicontinuous at $(\bar{y}, \bar{x})$ w.r.t. $\operatorname{dom} M$ while (FOSCclm) holds at this point, then $M$ is inner calm in the fuzzy sense at $(\bar{y}, \bar{x})$ w.r.t. $\operatorname{dom} M$. 


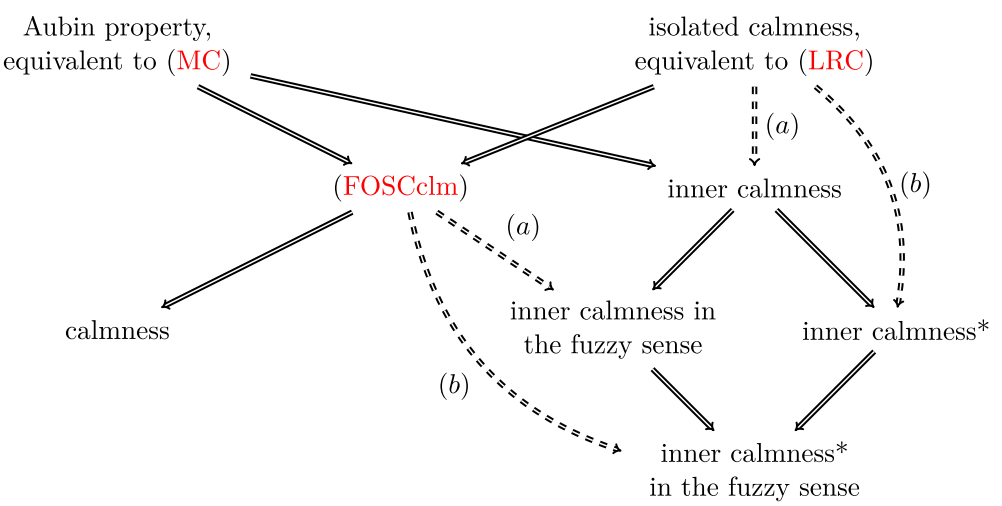

Fig. 1 Relations between the calmness-type conditions for mappings with a closed graph. Dashed relations only hold under additional assumptions in general. The additional assumptions $(a)$ and $(b)$ stand for inner semicontinuity and inner semicompactness, respectively, see Lemma 4.3 and Corollary 4.9

(ii) Let $M$ be inner semicompact at $\bar{y}$ w.r.t. $\operatorname{dom} M$ and let (FOSCclm) be valid at $(\bar{y}, \bar{x})$ for each $\bar{x} \in M(\bar{y})$. Then $M$ is inner calm* at $\bar{y}$ w.r.t. $\operatorname{dom} M$ in the fuzzy sense.

Proof Note that by inner semicompactness of $M$ at $\bar{y}$ w.r.t. $\operatorname{dom} M, \operatorname{dom} M$ is locally closed at $\bar{y}$ in both situations.

Let us start with the proof of (i). Inspecting Theorem 4.1, it is easy to show that $M$ is inner calm at $(\bar{y}, \bar{x})$ w.r.t. $\operatorname{dom} M$ in the fuzzy sense if and only if

$$
v \in T_{\operatorname{dom} M}(\bar{y}) \cap \mathcal{S} \Longrightarrow \inf _{u \in D M(\bar{y}, \bar{x})(v)}\|u\| /\|v\|<\infty
$$

is valid. Similar arguments as in the proof of Theorem 4.8 can be used in order to infer that $T_{\operatorname{dom} M}(\bar{y})=\operatorname{dom} D M(\bar{y}, \bar{x})$ holds due to the postulated inner semicontinuity of $M$ at $(\bar{y}, \bar{x})$ w.r.t. $\operatorname{dom} M$ and validity of (FOSCclm) at this point. Thus, the statement follows. For the proof of (ii), due to Theorem 4.1 and assertion (i) of Theorem 4.8, it remains to show

$$
v \in \bigcup_{\bar{x} \in M(\bar{y})} \operatorname{dom} D M(\bar{y}, \bar{x}) \cap \mathcal{S} \Longrightarrow \inf _{\bar{x} \in M(\bar{y})} \inf _{u \in D M(\bar{y}, \bar{x})(v)}\|u\| /\|v\|<\infty .
$$

The latter, however, is obvious.

In Fig. 1, we summarize the findings of this section regarding the relationship between all the introduced calmness-type conditions.

It remains an open question whether the validity of (FOSCclm) together with inner semicompactness of the underlying mapping is enough to already yield inner calmness*. At the moment, we are not aware of a counterexample.

\section{Some Calculus Rules}

In this section, we show how the two theorems in Section 3 translate into standard calculus rules. For brevity, in the first part, where we address standard calculus rules related to elementary set operations, we provide only outlines with essential information, and we forgo presenting actual formulas and other details. Later, when we deal with generalized 
derivatives of marginal functions as well as chain and product rules for the generalized differentiation of set-valued mappings, we present a detailed exposition.

\subsection{Calculus for Sets}

We consider four prototypical types of sets arising from elementary transformations, whose tangents and normals are to be computed: sums and intersections of closed sets as well as images and pre-images of closed sets under continuous transformations. Here, the main purpose is to show the connection to the theorems in Section 3 as well as to emphasize the structural similarities within these four prototypes - see e.g. the respective sufficient conditions. Note, however, that for the sum rule and the image rule, based on Theorem 3.1 , the estimates for tangents and regular normals, as well as the corresponding sufficient conditions are new (tangents to the image sets appeared in [4, Theorem 4.1, Corollary 4.2]). More precisely, we get equalities under fuzzy inner calmness*, which seems less restrictive than the corresponding convexity-based assumptions in [46], see also the comments after [4, Corollary 4.2].

In each case, the desired formulas can be derived by computing derivatives of a problemtailored associated set-valued mapping. We always hint how this can be done by means of the two elementary results from Lemmas 2.1 and 2.2. On top of it, we also comment on the closedness assumptions of the relevant theorem from Section 3, refer to the analogous estimates in [46], and, finally, provide sufficient conditions for the crucial calmness-type assumption.

\subsubsection{Sum Rule}

First, we consider the sum rule based on Theorem 3.1.

input: $\quad$ closed sets $D_{1}, \ldots, D_{\ell} \subset \mathbb{R}^{m}, D:=D_{1}+\ldots+D_{\ell}$;

associated mapping: $\quad M_{1}: \mathbb{R}^{m} \rightrightarrows\left(\mathbb{R}^{m}\right)^{\ell}$, given by

$$
M_{1}(y):=\left\{\left(y_{1}, \ldots, y_{\ell}\right) \in D_{1} \times \ldots \times D_{\ell} \mid y_{1}+\ldots+y_{\ell}=y\right\},
$$

satisfies $\operatorname{dom} M_{1}=D$ and

$$
\begin{aligned}
\operatorname{gph} M_{1}= & \left\{\left(y,\left(y_{1}, \ldots, y_{\ell}\right)\right) \mid\left(y_{1}, \ldots, y_{\ell}, y-y_{1}-\ldots-y_{\ell}\right)\right. \\
& \left.\times \in D_{1} \times \ldots \times D_{\ell} \times\{0\}\right\}
\end{aligned}
$$

closedness: $\quad$ gph $M_{1}$ closed by closedness of $D_{1}, \ldots, D_{\ell}$, dom $M_{1}$ closed by assumption or by the inner semicompactness of $M_{1}$ (e.g. if all except at most one of the sets $D_{1}, \ldots, D_{\ell}$ are bounded and, thus, compact);

derivatives of $M_{1}$ : use Lemmas 2.1 and 2.2;

estimates: similar to [46, Exercise 6.44], estimates for tangents can be enriched using bounds in terms of the modulus of fuzzy inner calmness*;

sufficient conditions: isolated calmness of $M_{1}$ at $\left(\bar{y},\left(\bar{y}_{1}, \ldots, \bar{y}_{\ell}\right)\right) \in \operatorname{gph} M_{1}$ (implied by):

$$
\left.\begin{array}{l}
v_{1}+\ldots+v_{\ell}=0 \\
v_{i} \in T_{D_{i}}\left(\bar{y}_{i}\right) i=1, \ldots, \ell
\end{array}\right\} \quad \Longrightarrow \quad v_{1}=\ldots=v_{\ell}=0
$$


FOSCclm for $M_{1}$ at $\left(\bar{y},\left(\bar{y}_{1}, \ldots, \bar{y}_{\ell}\right)\right)$ (implied by):

$$
\left.\begin{array}{l}
v_{1}+\ldots+v_{\ell}=0, \\
v_{i} \in T_{D_{i}}\left(\bar{y}_{i}\right) i=1, \ldots, \ell, \\
\left(v_{1}, \ldots, v_{\ell}\right) \neq(0, \ldots, 0)
\end{array}\right\} \Longrightarrow N_{D_{1}}\left(\bar{y}_{1} ; v_{1}\right) \cap \ldots \cap N_{D_{\ell}}\left(\bar{y}_{\ell} ; v_{\ell}\right)=\{0\}
$$

\subsubsection{Intersection Rule}

Second, we consider the intersection rule based on Theorem 3.2.

input: $\quad$ closed sets $C_{1}, \ldots, C_{\ell} \subset \mathbb{R}^{n}, C:=\bigcap_{i=1}^{\ell} C_{i}$;

associated mapping: $\quad M_{2}:\left(\mathbb{R}^{n}\right)^{\ell} \rightrightarrows \mathbb{R}^{n}$, given by

$$
\begin{aligned}
M_{2}\left(x_{1}, \ldots, x_{\ell}\right):= & \bigcap_{i=1}^{\ell}\left(C_{i}-x_{i}\right)=\{x \mid(x, \ldots, x) \\
& \left.+\left(x_{1}, \ldots, x_{\ell}\right) \in C_{1} \times \ldots \times C_{\ell}\right\},
\end{aligned}
$$

satisfies $M_{2}(0, \ldots, 0)=C$ and

$$
\operatorname{gph} M_{2}=\left\{\left(\left(x_{1}, \ldots, x_{\ell}\right), x\right) \mid(x, \ldots, x)+\left(x_{1}, \ldots, x_{\ell}\right) \in C_{1} \times \ldots \times C_{\ell}\right\} ;
$$

closedness: $\quad$ gph $M_{2}$ closed by closedness of $C_{1}, \ldots, C_{\ell}$;

derivatives of $M_{2}$ : use Lemmas 2.1 and 2.2;

estimates: similar to [46, Theorem 6.42], estimates for (directional) limiting normals can be enriched using bounds in terms of the (directional) calmness modulus;

sufficient conditions: Aubin property of $M_{2}$ at $((0, \ldots, 0), \bar{x}) \in \operatorname{gph} M_{2}$ :

$$
\left.\begin{array}{l}
x_{1}^{*}+\ldots+x_{\ell}^{*}=0, \\
x_{i}^{*} \in N_{C_{i}}(\bar{x}) i=1, \ldots, \ell
\end{array}\right\} \quad \Longrightarrow \quad x_{1}^{*}=\ldots=x_{\ell}^{*}=0
$$

FOSCclm for $M_{2}$ at $((0, \ldots, 0), \bar{x})$ (implied by):

$$
\left.\begin{array}{l}
x_{1}^{*}+\ldots+x_{\ell}^{*}=0, \\
x_{i}^{*} \in N_{C_{i}}(\bar{x} ; u) i=1, \ldots, \ell, \\
u \neq 0
\end{array}\right\} \Longrightarrow x_{1}^{*}=\ldots=x_{\ell}^{*}=0
$$

\subsubsection{Image Rule}

Third, we consider the image rule based on Theorem 3.1.

input: continuous mapping $g: \mathbb{R}^{n} \rightarrow \mathbb{R}^{m}$, closed set $C \subset \mathbb{R}^{n}, D:=g(C)$; associated mapping: $\quad M_{3}: \mathbb{R}^{m} \rightrightarrows \mathbb{R}^{n}$, given by

$$
M_{3}(y):=g^{-1}(y) \cap C,
$$

satisfies $\operatorname{dom} M_{3}=D$ and gph $M_{3}=\operatorname{gph} g^{-1} \cap\left(\mathbb{R}^{m} \times C\right)$;

closedness: $\quad$ ph $M_{3}$ closed by closedness of $C$ and continuity of $g$, $\operatorname{dom} M_{3}$ closed (locally around $\bar{y}$ ) by assumption or by the inner semicompactness of $M_{3}$ (e.g. if $g^{-1}(V) \cap C$ is bounded for some neighborhood $V$ of $\bar{y}$ );

derivatives of $M_{3}$ : use the above intersection rule, justified e.g. if $g$ is locally Lipschitzian at $\bar{x} \in M_{3}(\bar{y})$, since (5.2) reads as $D^{*} g(\bar{x})(0) \cap\left(-N_{C}(\bar{x})\right)=\{0\}$;

estimates: similar to [46, Theorem 6.43], estimates for tangents can be enriched using bounds in terms of the modulus of fuzzy inner calmness*; 
sufficient conditions: isolated calmness of $M_{3}$ at $(g(\bar{x}), \bar{x}) \in \operatorname{gph} M_{3}$ (implied by):

$$
0 \in D g(\bar{x})(u), u \in T_{C}(\bar{x}) \quad \Longrightarrow \quad u=0 ;
$$

FOSCclm for $M_{3}$ at $(g(\bar{x}), \bar{x})$ (implied by):

$$
D^{*} g(\bar{x} ;(u, 0))\left(y^{*}\right) \cap\left(-N_{C}(\bar{x} ; u)\right) \neq \varnothing, u \neq 0 \quad \Longrightarrow \quad y^{*}=0
$$

\subsubsection{Pre-Image Rule}

Fourth, we consider the pre-image rule based on Theorem 3.2.

input: continuous mapping $g: \mathbb{R}^{n} \rightarrow \mathbb{R}^{m}$, closed set $D \subset \mathbb{R}^{m}, C:=g^{-1}(D)$; associated mapping: $\quad M_{4}: \mathbb{R}^{m} \rightrightarrows \mathbb{R}^{n}$, given by

$$
M_{4}(y):=\{x \mid g(x)+y \in D\},
$$

satisfies $M_{4}(0)=C$ and gph $M_{4}=\operatorname{gph}(-g)^{-1}+(D \times\{0\})$;

closedness: $\quad$ gph $M_{4}$ closed by closedness of $D$ and continuity of $g$;

derivatives of $M_{4}$ : use the above sum rule, justified e.g. if $g$ is calm at $\bar{x}$ where $\bar{x} \in$ $M_{4}(0)$, since (5.1) reads as $D g(\bar{x})(0) \cap T_{D}(g(\bar{x}))=\{0\}$;

estimates: similar to [46, Theorem 6.14, Theorem 6.31], estimates for (directional) limiting normals can be enriched using bounds in terms of the (directional) calmness modulus;

sufficient conditions: Aubin property of $M_{4}$ at $(0, \bar{x}) \in \operatorname{gph} M_{4}$ :

$$
0 \in D^{*} g(\bar{x})\left(y^{*}\right), y^{*} \in N_{D}(g(\bar{x})) \Longrightarrow y^{*}=0 ;
$$

FOSCclm for $M_{4}$ at $(0, \bar{x})$ (implied by):

$$
0 \in D^{*} g(\bar{x} ;(u, v))\left(y^{*}\right), y^{*} \in N_{D}(g(\bar{x}) ; v), u \neq 0 \Longrightarrow y^{*}=0
$$

\subsection{Derivatives of Marginal Functions}

On the basis of the two patterns from Section 3, one could derive the majority of calculus rules for subderivatives and subdifferentials, such as the sum rule and the chain rule. These two are, however, based on Theorem 3.2 and well-understood, so our approach provides little novelty there. Hence, we only focus here on one prominent rule related to Theorem 3.1 .

To this end, we fix a lower semicontinuous, extended real-valued function $f: \mathbb{R}^{n} \times$ $\mathbb{R}^{m} \rightarrow \overline{\mathbb{R}}$ and consider the associated marginal (or optimal value) function $\vartheta: \mathbb{R}^{m} \rightarrow \overline{\mathbb{R}}$ as well as the associated solution mapping $S: \mathbb{R}^{m} \rightrightarrows \mathbb{R}^{n}$ given by

$$
\vartheta(y):=\inf _{x} f(x, y) \quad S(y):=\underset{x}{\operatorname{argmin}} f(x, y),
$$

respectively. Let $\bar{y} \in \operatorname{dom} S$ be chosen such that $\vartheta(\bar{y})$ is finite.

In [30, Propositions 3.2 and 3.3.], the authors provide a direct proof of upper estimates for the limiting subdifferential of $\vartheta$ at $\bar{y}$ under the assumption that the solution map $S$ is inner semicontinuous at some point $(\bar{y}, \bar{x}) \in \operatorname{gph} S$ or inner semicompact at $\bar{y}$, respectively. In [46, Theorem 10.13], upper estimates for the regular and the limiting subdifferential of $\vartheta$ at $\bar{y}$ are derived via the image rule while exploiting that epi $\vartheta$ can be interpreted as a projection of epi $f$ under mild assumptions. In the light of our refined image rule from Section 5.1, one has to impose some inner semicompactness or inner calmness* (in the fuzzy sense) of the mapping

$$
\Psi(y, \alpha):=\left(\mathbb{R}^{n} \times\{(y, \alpha)\}\right) \cap \text { epi } f
$$


at $(\bar{y}, \vartheta(\bar{y}))$ for that purpose.

Here, we strike a slightly different path. Let us consider the problem-tailored level set mapping $M: \mathbb{R}^{m} \times \mathbb{R} \rightrightarrows \mathbb{R}^{n}$ given by

$$
M(y, \alpha):=\left\{x \in \mathbb{R}^{n} \mid f(x, y) \leq \alpha\right\}=\left\{x \in \mathbb{R}^{n} \mid(x, y, \alpha) \in \text { epi } f\right\} .
$$

By definition, we have $\operatorname{dom} M=$ epi $\vartheta$ and gph $M=\pi^{-1}$ (epi $f$ ), where $\pi(y, \alpha, x)=$ $(x, y, \alpha)$ merely permutes the variables, so there are trivial relations between derivatives of $M$ and $f$, see Lemma 2.1. Since $\Psi(y, \alpha)=(M(y, \alpha),(y, \alpha))$, by Proposition 2.6, we see that it is the same whether we apply the corresponding assumptions on $M$ or $\Psi$, see also Remark 3.3. On the other hand, we have $S(y)=M(y, \vartheta(y))$. Thus, the comparison of the assumptions imposed on $S$ and $M$ via Proposition 2.6 seems to be a more delicate issue depending on the properties of $\vartheta$, which is an interesting topic on its own, see [34]. A direct comparison (between $S$ and $\Psi$ ), however, was performed in [6, Proof of Theorem 4.2].

Theorem 3.1 yields the following estimates for the generalized derivatives of $\vartheta$.

Theorem 5.1 Fix $\bar{y} \in \operatorname{dom} S$ where $\vartheta$ is finite and $\operatorname{dom} S$ is locally closed. Then the following assertions hold.

d Subderivative: For each direction $v \in \mathbb{R}^{m}$ and $\kappa>0$, we always have

$$
\mathrm{d} \vartheta(\bar{y})(v) \leq \inf _{\bar{x} \in S(\bar{y})}\left(\inf _{u \in \kappa\|(v, \mathrm{~d} \vartheta(\bar{y})(v))\| \mathcal{B}} \mathrm{d} f(\bar{x}, \bar{y})(u, v)\right),
$$

and the opposite inequality holds true if $M$ is inner calm* at $(\bar{y}, \vartheta(\bar{y}))$ w.r.t. $\operatorname{dom} M$ in direction $(v, \mathrm{~d} \vartheta(\bar{y})(v))$ in the fuzzy sense with modulus smaller than $\kappa$.

$\widehat{\partial} \quad$ Regular subdifferential: We always have

$$
y^{*} \in \widehat{\partial} \vartheta(\bar{y}) \Longrightarrow\left(0, y^{*}\right) \in \bigcap_{\bar{x} \in S(\bar{y})} \widehat{\partial} f(\bar{x}, \bar{y}),
$$

and the opposite implication holds true if $M$ is inner calm* at $(\bar{y}, \vartheta(\bar{y}))$ w.r.t. $\operatorname{dom} M$ in the fuzzy sense.

d Limiting subdifferential: If $M$ is inner semicompact at $(\bar{y}, \vartheta(\bar{y}))$ w.r.t. $\operatorname{dom} M$, then we have

$$
y^{*} \in \partial \vartheta(\bar{y}) \Longrightarrow\left(0, y^{*}\right) \in \bigcup_{\bar{x} \in S(\bar{y})} \partial f(\bar{x}, \bar{y}) .
$$

d2 Directional limiting subdifferential: If $M$ is inner calm* at $(\bar{y}, \vartheta(\bar{y}))$ in direction $(v, \mu) \in e p i \mathrm{~d} \vartheta(\bar{y})$ w.r.t. $\operatorname{dom} M$, we have

$$
y^{*} \in \partial \vartheta(\bar{y} ;(v, \mu)) \Longrightarrow\left(0, y^{*}\right) \in \bigcup_{\bar{x} \in S(\bar{y})} \bigcup_{(u, v, \mu) \in e p i \mathrm{~d} f(\bar{x}, \bar{y})} \partial f((\bar{x}, \bar{y}) ;(u, v, \mu)) .
$$

Proof Let us start proving the relations for the subderivative. Taking into account that tangents to the epigraph of a function are precisely elements of the epigraph of its subderivative, Theorem 3.1 yields

$$
\mu \geq \mathrm{d} \vartheta(\bar{y})(v) \Longleftarrow \exists \bar{x} \in S(\bar{y}), \exists u \in \kappa\|(v, \mu)\| \mathcal{B}: \mu \geq \mathrm{d} f(\bar{x}, \bar{y})(u, v)
$$

for arbitrary $\kappa>0$, and the opposite implication holds true if $M$ is inner calm* at $(\bar{y}, \vartheta(\bar{y}))$ w.r.t. dom $M$ in direction $(v, \mu)$ in the fuzzy sense with modulus smaller than $\kappa$.

Next, given any $\varepsilon>0$, there exist $\bar{x}_{\varepsilon} \in S(\bar{y})$ and $u_{\varepsilon} \in \kappa\|(v, \mathrm{~d} \vartheta(\bar{y})(v))\| \mathcal{B}$ such that

$$
\mathrm{d} f\left(\bar{x}_{\varepsilon}, \bar{y}\right)\left(u_{\varepsilon}, v\right) \leq \inf _{\bar{x} \in S(\bar{y})}\left(\inf _{u \in \kappa\|(v, \mathrm{~d} \vartheta(\bar{y})(v))\| \mathcal{B}} \mathrm{d} f(\bar{x}, \bar{y})(u, v)\right)+\varepsilon .
$$




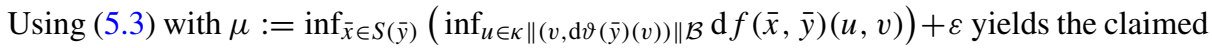
inequality as $\varepsilon \downarrow 0$. Note that $u_{\varepsilon} \in \kappa\|(v, \mu)\| \mathcal{B}$ may not be satisfied, but this is not a problem since (5.3) holds also without the bound on $u$.

On the other hand, if $M$ is inner calm* at $(\bar{y}, \vartheta(\bar{y}))$ w.r.t. dom $M$ in direction $(v, \mathrm{~d} \vartheta(\bar{y})(v))$ in the fuzzy sense, the forward implication in (5.3) with $\mu:=\mathrm{d} \vartheta(\bar{y})(v)$ yields the estimate $\mathrm{d} \vartheta(\bar{y})(v) \geq \mathrm{d} f(\bar{x}, \bar{y})(u, v)$ for some $\bar{x} \in S(\bar{y})$ and $u \in \kappa\|(v, \mathrm{~d} \vartheta(\bar{y})(v))\| \mathcal{B}$, and we have equality in the formula for the subderivative.

The subdifferential estimates follow directly from the definitions provided in Section 2.1 as well as Theorem 3.1.

While statement $\partial$ is a standard result and statement $\mathbf{d} \partial$ is an easy extension of [6, Theorem 4.2 ], the estimates from statements $\mathbf{d}$ and $\widehat{\partial}$, based on fuzzy inner calmness*, are new.

Moreover, based on the results from Section 4, we can exploit the following sufficient conditions in order to guarantee the validity of the inner calmness* type assumptions which appear in Theorem 5.1. Using the Levy-Rockafellar criterion, the isolated calmness of $M$ at $((\bar{y}, \vartheta(\bar{y})), \bar{x}) \in \operatorname{gph} M$ reads as

$\mathrm{d} f(\bar{x}, \bar{y})(u, 0) \leq 0 \Longrightarrow u=0 \Longleftrightarrow \mathrm{d} f(\bar{x}, \bar{y})(u, 0)>0 \forall(u, 0) \in \operatorname{dom} \mathrm{d} f(\bar{x}, \bar{y}), u \neq 0$,

while FOSCclm for $M$ at $((\bar{y}, \vartheta(\bar{y})), \bar{x})$ is given by

$$
\begin{aligned}
\forall u \in \mathbb{R}^{n} \backslash\{0\}: \quad \mathrm{d} f(\bar{x}, \bar{y})(u, 0) \leq 0 \\
\Longrightarrow \quad\left\{\begin{array}{l}
\left(0, y^{*}\right) \notin \partial f((\bar{x}, \bar{y}) ;(u, 0,0)) \quad \forall y^{*} \in \mathbb{R}^{m}, \\
\left(0, y^{*}\right) \in \partial^{\infty} f((\bar{x}, \bar{y}) ;(u, 0,0)) \Longrightarrow y^{*}=0 .
\end{array}\right.
\end{aligned}
$$

The latter claim deserves a justification. Suppose (5.4) holds and observe

$$
\begin{aligned}
\left(y^{*}, \lambda^{*}\right) & \in D^{*} M(((\bar{y}, \vartheta(\bar{y})), \bar{x}) ;((0,0), u))(0) \\
& \Longleftrightarrow \quad\left(0, y^{*}, \lambda^{*}\right) \in N_{\text {epi } f}(((\bar{x}, \bar{y}), f(\bar{x}, \bar{y})) ;((u, 0), 0)) .
\end{aligned}
$$

Hence, $\lambda^{*} \leq 0$ holds. If $\lambda^{*}<0$ is valid, we get $\left(0,-y^{*} / \lambda^{*}\right) \in \partial f((\bar{x}, \bar{y}) ;(u, 0,0))$ which contradicts (5.4) and so $\lambda^{*}=0$. Then, however, we obtain $\left(0, y^{*}\right) \in \partial^{\infty} f((\bar{x}, \bar{y}) ;(u, 0,0))$ and $y^{*}=0$ follows. This proves FOSCclm for $M$ at $((\bar{y}, \vartheta(\bar{y})), \bar{x})$. The reverse implication is now clear as well.

Finally, note that the weaker condition

$\forall u \in \mathbb{R}^{n} \backslash\{0\}: \quad \mathrm{d} f(\bar{x}, \bar{y})(u, 0) \leq 0 \Longrightarrow\left[\left(0, y^{*}\right) \in \partial f((\bar{x}, \bar{y}) ;(u, 0,0)) \Longrightarrow y^{*}=0\right]$

can be used here the same way as FOSCclm and isolated calmness. Indeed, one can derive from Theorem 3.1 the corresponding estimate for directional subdifferentials, valid under just inner semicompactness of $M$ and employing directions of the form $(u, 0,0)$, and then observe that (5.5) also makes the additional union superfluous. Then the same arguments as those in the proof of Theorem 4.8, that justified FOSCclm, apply here as well. 


\subsection{Chain Rule for Set-Valued Mappings}

Let us consider set-valued mappings $S_{1}: \mathbb{R}^{n} \rightrightarrows \mathbb{R}^{m}$ and $S_{2}: \mathbb{R}^{m} \rightrightarrows \mathbb{R}^{\ell}$ with closed graphs as well as their composition $S_{2} \circ S_{1}: \mathbb{R}^{n} \rightrightarrows \mathbb{R}^{\ell}$ given by

$$
\left(S_{2} \circ S_{1}\right)(x):=\bigcup_{y \in S_{1}(x)} S_{2}(y) .
$$

For later use, we introduce $S: \mathbb{R}^{n} \rightrightarrows \mathbb{R}^{\ell}$ as $S:=S_{2} \circ S_{1}$. Furthermore, let $\Xi: \mathbb{R}^{n} \times \mathbb{R}^{\ell} \rightrightarrows \mathbb{R}^{m}$ denote the standard "intermediate" mapping given by

$$
\Xi(x, z):=S_{1}(x) \cap S_{2}^{-1}(z)=\left\{y \in S_{1}(x) \mid z \in S_{2}(y)\right\} .
$$

In [46, Theorem 10.37], the authors use a combination of the image rule and the preimage rule in order to compute the limiting coderivative of the composition $S$. On the other hand, in [30, Section 7], the authors prove the chain rule for the limiting coderivative via subdifferentials, namely a combination of the rule for the marginal function and the sum rule. In [41, Theorem 3.11], the author exploits the sum rule for the limiting coderivative in order to derive the chain rule for the latter.

As before, we provide a different approach utilizing our results from Theorems 3.1 and 3.2. To this end, we introduce the perturbation map $M: \mathbb{R}^{m} \times \mathbb{R}^{\ell} \rightrightarrows \mathbb{R}^{n} \times \mathbb{R}^{\ell} \times \mathbb{R}^{m}$ given by

$$
M(p, q):=\left\{(x, z, y) \mid y+p \in S_{1}(x), z+q \in S_{2}(y)\right\},
$$

and we point out the relations

$$
\operatorname{gph} S=\operatorname{dom} \Xi \quad \operatorname{gph} \Xi=M(0,0) .
$$

Clearly, the results from Theorems 3.1 and 3.2 can be used to establish a connection between the derivatives of $S$ and $M$ (not $S_{1}$ and $S_{2}$ ). Additionally, observing that

$$
\operatorname{gph} M=\left\{((p, q),(x, z, y)) \mid((x, y+p),(y, z+q)) \in \operatorname{gph} S_{1} \times \operatorname{gph} S_{2}\right\}
$$

holds, we readily infer for arbitrary $((\bar{x}, \bar{z}), \bar{y}) \in \operatorname{gph} \Xi$ the relations

$$
D M((0,0),(\bar{x}, \bar{z}, \bar{y}))(0,0) \subset\left\{(u, w, v) \mid v \in D S_{1}(\bar{x}, \bar{y})(u), w \in D S_{2}(\bar{y}, \bar{z})(v)\right\},
$$

and

$$
\begin{gathered}
\widehat{D}^{*} M((0,0),(\bar{x}, \bar{z}, \bar{y}))\left(-x^{*}, z^{*}, 0\right) \\
=\left\{\left(-p^{*},-z^{*}\right) \mid x^{*} \in \widehat{D}^{*} S_{1}(\bar{x}, \bar{y})\left(p^{*}\right), p^{*} \in \widehat{D}^{*} S_{2}(\bar{y}, \bar{z})\left(z^{*}\right)\right\}
\end{gathered}
$$

from Lemmas 2.1 and 2.2, and analogous estimates are valid for the limiting and the directional limiting coderivate (with relations $=$ and $\subset$, respectively). Moreover, all inclusions become equalities under the following equivalence:

$$
\begin{aligned}
((u, v),(v, w)) & \in T_{\operatorname{gph} S_{1} \times \operatorname{gph} S_{2}}((\bar{x}, \bar{y}),(\bar{y}, \bar{z})) \\
& \Longleftrightarrow v \in D S_{1}(\bar{x}, \bar{y})(u), w \in D S_{2}(\bar{y}, \bar{z})(v) .
\end{aligned}
$$

Putting all these things together, we arrive at the following result.

Theorem 5.2 Fix $(\bar{x}, \bar{z}) \in \operatorname{gph} S$ where $\operatorname{gph} S$ is locally closed. Then the following assertions hold.

D Graphical derivative: If $\Xi$ is inner calm* at $(\bar{x}, \bar{z})$ w.r.t. dom $\Xi$ in the fuzzy sense, then

$$
D S(\bar{x}, \bar{z})(u) \subset \bigcup_{\bar{y} \in \Xi(\bar{x}, \bar{z})}\left(D S_{2}(\bar{y}, \bar{z}) \circ D S_{1}(\bar{x}, \bar{y})\right)(u) .
$$


The opposite inclusion holds true if, for all $\bar{y} \in \Xi(\bar{x}, \bar{z}), M$ is calm at $((0,0)$, $(\bar{x}, \bar{z}, \bar{y}))$ while (5.9) is valid.

$\widehat{\mathbf{D}}^{*} \quad$ Regular coderivative: If $\Xi$ is inner calm* at $(\bar{x}, \bar{z})$ w.r.t. dom $\Xi$ in the fuzzy sense, then

$$
\widehat{D}^{*} S(\bar{x}, \bar{z})\left(z^{*}\right) \supset \bigcap_{\bar{y} \in \Xi(\bar{x}, \bar{z})}\left(\widehat{D}^{*} S_{1}(\bar{x}, \bar{y}) \circ \widehat{D}^{*} S_{2}(\bar{y}, \bar{z})\right)\left(z^{*}\right) .
$$

On the other hand, if $M$ is calm at each point $((0,0),(\bar{x}, \bar{z}, \bar{y}))$ such that $\bar{y} \in$ $\Xi(\bar{x}, \bar{z})$, then we also have the following upper estimate in terms of the limiting coderivative:

$$
\widehat{D}^{*} S(\bar{x}, \bar{z})\left(z^{*}\right) \subset \bigcap_{\bar{y} \in \Xi(\bar{x}, \bar{z})}\left(D^{*} S_{1}(\bar{x}, \bar{y}) \circ D^{*} S_{2}(\bar{y}, \bar{z})\right)\left(z^{*}\right) .
$$

$\mathbf{D}^{*} \quad$ Limiting coderivative: If $\Xi$ is inner semicompact at $(\bar{x}, \bar{z})$ w.r.t. $\operatorname{dom} \Xi$ and if $M$ is calm at each point $((0,0),(\bar{x}, \bar{z}, \bar{y}))$ such that $\bar{y} \in \Xi(\bar{x}, \bar{z})$, then

$$
D^{*} S(\bar{x}, \bar{z})\left(z^{*}\right) \subset \bigcup_{\bar{y} \in \Xi(\bar{x}, \bar{z})}\left(D^{*} S_{1}(\bar{x}, \bar{y}) \circ D^{*} S_{2}(\bar{y}, \bar{z})\right)\left(z^{*}\right) .
$$

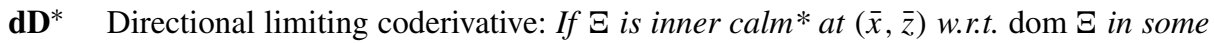
direction $(u, w) \in \mathbb{R}^{n} \times \mathbb{R}^{\ell}$ and if $M$ is calm at each point $((0,0),(\bar{x}, \bar{z}, \bar{y}))$ with $\bar{y} \in \Xi(\bar{x}, \bar{z})$ and in each direction $(u, w, v)$ with $v \in D \Xi((\bar{x}, \bar{z}), \bar{y})(u, w)$, then

$$
\begin{aligned}
& D^{*} S((\bar{x}, \bar{z}) ;(u, w))\left(z^{*}\right) \\
& \quad \subset \bigcup_{\bar{y} \in \Xi(\bar{x}, \bar{z})} \bigcup_{v \in D \Xi((\bar{x}, \bar{z}), \bar{y})(u, w)}\left(D^{*} S_{1}((\bar{x}, \bar{y}) ;(u, v)) \circ D^{*} S_{2}((\bar{y}, \bar{z}) ;(v, w))\right)\left(z^{*}\right) .
\end{aligned}
$$

Proof The proof in fact follows easily from the relations (5.8), Theorems 3.1 and 3.2, and the above formulas for derivatives of $M$.

Let us start to prove the assertions from $\mathbf{D}$. Therefore, fix $w \in D S(\bar{x}, \bar{z})(u)$ for some arbitrary $u \in \mathbb{R}^{n}$. Due to gph $S=\operatorname{dom} \Xi$ and the fuzzy inner calmness* of $\Xi$ at $(\bar{x}, \bar{z})$ w.r.t. dom $\Xi$, we find $\bar{y} \in \Xi(\bar{x}, \bar{z})$ and $v \in D \Xi((\bar{x}, \bar{z}), \bar{y})(u, w)$ by Theorem 3.1. Due to gph $\Xi=M(0,0)$, we have $((u, w), v) \in T_{M(0,0)}((\bar{x}, \bar{z}), \bar{y})$. Now, we can apply Theorem 3.2 in order to find $(u, w, v) \in D M((0,0),(\bar{x}, \bar{z}, \bar{y}))(0,0)$, and this yields $w \in\left(D S_{2}(\bar{y}, \bar{z}) \circ\right.$ $\left.D S_{1}(\bar{x}, \bar{y})\right)(u)$. The opposite inclusion follows by the same steps in reverse order while noting that equality holds in the formula for the graphical derivative for $M$ due to (5.9) and that the calmness of $M$ at all reference points is needed in order to obtain equality in the first assertion of Theorem 3.2.

Next, we investigate the assertions from $\widehat{\mathbf{D}}^{*}$. Fix an arbitrary $\bar{y} \in \Xi(\bar{x}, \bar{z})$ as well as some $x^{*} \in\left(\widehat{D}^{*} S_{1}(\bar{x}, \bar{y}) \circ \widehat{D}^{*} S_{2}(\bar{y}, \bar{z})\right)\left(z^{*}\right)$ for arbitrarily chosen $z^{*} \in \mathbb{R}^{\ell}$, i.e., $\left(-x^{*}, z^{*}, 0\right) \in$ $\operatorname{dom} \widehat{D}^{*} M((0,0),(\bar{x}, \bar{z}, \bar{y}))$. Due to gph $\Xi=M(0,0)$, Theorem 3.2 applies here and yields $\left(x^{*},-z^{*}\right) \in \widehat{D}^{*} \Xi((\bar{x}, \bar{z}), \bar{y})(0)$. Observing that this holds for all $\bar{y} \in \Xi(\bar{x}, \bar{z})$ while $\Xi$ is assumed to be inner calm* at $(\bar{x}, \bar{z})$ w.r.t. dom $\Xi$ in the fuzzy sense, Theorem 3.1 leads to $\left(x^{*},-z^{*}\right) \in \widehat{N}_{\text {dom } \Xi}(\bar{x}, \bar{z})$. Since dom $\Xi=\operatorname{gph} S$ is valid, we have shown the validity of the lower estimate for the regular coderivative.

On the other hand, given $x^{*} \in \widehat{D}^{*} S(\bar{x}, \bar{z})\left(z^{*}\right)$ for arbitrary $z^{*} \in \mathbb{R}^{\ell}$, Theorem 3.1 implies $\left(x^{*},-z^{*}\right) \in \widehat{D}^{*} \Xi((\bar{x}, \bar{z}), \bar{y})(0)$ for each $\bar{y} \in \Xi(\bar{x}, \bar{z})$. Replacing the regular coderivative by the limiting one and utilizing the assumed calmness of $M$, Theorem 3.2 leads to $\left(-x^{*}, z^{*}, 0\right) \in \operatorname{dom} D^{*} M((0,0),(\bar{x}, \bar{z}, \bar{y}))$ and the upper estimate for the regular coderivative follows. 
The remaining proofs for the assertions in $\mathbf{D}^{*}$ and $\mathbf{d} \mathbf{D}^{*}$ are analogous to the validation of the upper estimate for the regular coderivative.

Let us point out that the upper estimate for the regular coderivative via an intersection is generally tighter than the one for the limiting coderivative which is given in terms of a union. This is achieved by replacing the regular coderivative by the limiting one only after the first calculus step instead of doing it at the beginning.

Exploiting the precise moduli of fuzzy inner calmness* and (directional) calmness in the proof of Theorem 5.2, one obtains estimates which comprise bounds.

Corollary 5.3 Fix $(\bar{x}, \bar{z}) \in \operatorname{gph} S$ where gph $S$ is locally closed.

D Graphical derivative: If $\Xi$ is inner calm $^{*}$ at $(\bar{x}, \bar{z})$ w.r.t. $\operatorname{dom} \Xi$ in direction $(u, w)$ in the fuzzy sense with modulus smaller than $\kappa$, then

$$
\begin{aligned}
w & \in D S(\bar{x}, \bar{z})(u) \\
& \Longrightarrow \exists \bar{y} \in \Xi(\bar{x}, \bar{z}) \exists v \in D S_{1}(\bar{x}, \bar{y})(u) \cap \kappa\|(u, w)\| \mathcal{B}: w \in D S_{2}(\bar{y}, \bar{z})(v) .
\end{aligned}
$$

D* Limiting coderivative: If $\Xi$ is inner semicompact at $(\bar{x}, \bar{z})$ w.r.t. $\operatorname{dom} \Xi$ and if $M$ is calm at each point $((0,0),(\bar{x}, \bar{z}, \bar{y}))$ such that $\bar{y} \in \Xi(\bar{x}, \bar{z})$ with modulus smaller than $\kappa_{\bar{y}}$, then

$$
\begin{gathered}
x^{*} \in D^{*} S(\bar{x}, \bar{z})\left(z^{*}\right) \\
\Longrightarrow \exists \bar{y} \in \Xi(\bar{x}, \bar{z}) \exists y^{*} \in D^{*} S_{2}(\bar{y}, \bar{z})\left(z^{*}\right) \cap \kappa_{\bar{y}}\left\|\left(x^{*}, z^{*}\right)\right\| \mathcal{B}: x^{*} \in D^{*} S_{1}(\bar{x}, \bar{y})\left(y^{*}\right) .
\end{gathered}
$$

Naturally, the upper estimate for the regular coderivative and the estimate for the directional limiting coderivative can also be enriched by the (directional) calmness modulus.

In the subsequent remark, we comment on the graphical derivative of $\Xi$, which appears in $\mathbf{d D}^{*}$, as well as on the directional limiting coderivative of $\Xi$, which will be important later in the discussion about sufficient conditions for the calmness conditions appearing in Theorem 5.2.

Remark 5.4 Fix $(\bar{x}, \bar{z}) \in \operatorname{gph} S$ and $\bar{y} \in \Xi(\bar{x}, \bar{z})$ as well as a pair $(u, w) \in \mathbb{R}^{n} \times \mathbb{R}^{\ell}$. Due to gph $\Xi=M(0,0)$, we can apply Theorem 3.2 and the formula for the graphical derivative of $M$ in order to find the upper estimate

$$
D \Xi((\bar{x}, \bar{z}), \bar{y})(u, w) \subset D S_{1}(\bar{x}, \bar{y})(u) \cap D S_{2}(\bar{y}, \bar{z})^{-1}(w) .
$$

On the other hand, $v \in D S_{1}(\bar{x}, \bar{y})(u) \cap D S_{2}(\bar{y}, \bar{z})^{-1}(w)$ belongs to $D \Xi((\bar{x}, \bar{z}), \bar{y})(u, w)$ whenever $M$ is calm at $((0,0),(\bar{x}, \bar{z}, \bar{y}))$ while (5.9) holds. Moreover, directional calmness of $M$ at $((0,0),(\bar{x}, \bar{z}, \bar{y}))$ in direction $(u, w, v)$ yields

$$
\begin{aligned}
& \left(x^{*},-z^{*}\right) \in D^{*} \Xi(((\bar{x}, \bar{z}), \bar{y}) ;((u, w), v))(0) \\
\Longrightarrow & x^{*} \in\left(D^{*} S_{1}((\bar{x}, \bar{y}) ;(u, v)) \circ D^{*} S_{2}((\bar{y}, \bar{z}) ;(v, w))\right)\left(z^{*}\right) .
\end{aligned}
$$

Let us now comment on sufficient conditions for the presence of the additional calmness properties in Theorem 5.2. With the aid of the Mordukhovich criterion and the formulas for the derivatives of $M$, we can easily check that $M$ possesses the Aubin property at some point $((0,0),(\bar{x}, \bar{z}, \bar{y})) \in \mathrm{gph} M$ if and only if the condition

$$
\operatorname{ker} D^{*} S_{1}(\bar{x}, \bar{y}) \cap D^{*} S_{2}(\bar{y}, \bar{z})(0)=\{0\}
$$


holds. This is the essential qualification condition which appears frequently in the literature in order to guarantee the validity of the chain rule for the limiting coderivative, see e.g. [41, Theorem 3.9] or [46, Theorem 10.37]. Moreover, it is implied, in particular, if either $S_{1}$ is metrically regular at $(\bar{x}, \bar{y})$ or $S_{2}$ has the Aubin property at $(\bar{y}, \bar{z})$. Finally, FOSCclm of $M$ at $((0,0),(\bar{x}, \bar{z}, \bar{y}))$ holds if for all nonzero $(u, v, w)$ with $v \in D S_{1}(\bar{x}, \bar{y})(u)$ and $w \in D S_{2}(\bar{y}, \bar{z})(v)$ one has

$$
\operatorname{ker} D^{*} S_{1}((\bar{x}, \bar{y}) ;(u, v)) \cap D^{*} S_{2}((\bar{y}, \bar{z}) ;(v, w))(0)=\{0\} .
$$

On the other hand, on the basis of Remark 5.4 and the Levy-Rockafellar criterion, we infer that $\Xi$ is isolatedly calm at some point $((\bar{x}, \bar{z}), \bar{y}) \in$ gph $\Xi$ provided

$$
D S_{1}(\bar{x}, \bar{y})(0) \cap \operatorname{ker} D S_{2}(\bar{y}, \bar{z})=\{0\}
$$

holds. Particularly, this is satisfied if either $S_{1}$ is isolatedly calm at $(\bar{x}, \bar{y})$ or $S_{2}$ is strongly metrically subregular at $(\bar{y}, \bar{z})$. Moreover, FOSCclm for $\Xi$ holds at $((\bar{x}, \bar{z}), \bar{y})$ if one has

$$
D^{*} \Xi(((\bar{x}, \bar{z}), \bar{y}) ;((0,0), v))(0)=\{(0,0)\} \quad \forall v \in\left(D S_{1}(\bar{x}, \bar{y})(0) \cap \operatorname{ker} D S_{2}(\bar{y}, \bar{z})\right) \backslash\{0\} .
$$

Naturally, this can be secured in terms of $S_{1}$ and $S_{2}$ by

$$
x^{*} \in\left(D^{*} S_{1}((\bar{x}, \bar{y}) ;(0, v)) \circ D^{*} S_{2}((\bar{y}, \bar{z}) ;(v, 0))\right)\left(z^{*}\right) \Longrightarrow x^{*}, z^{*}=0,
$$

provided $M$ is calm at $((0,0),(\bar{x}, \bar{z}, \bar{y}))$ in direction $(0,0, v)$, see Remark 5.4 again.

Finally, note that the standard approach to the chain rule from the literature typically asks for calmness of the mapping $\tilde{M}: \mathbb{R}^{n} \times \mathbb{R}^{m} \times \mathbb{R}^{m} \times \mathbb{R}^{\ell} \rightrightarrows \mathbb{R}^{n} \times \mathbb{R}^{\ell} \times \mathbb{R}^{m}$ given by

$$
\tilde{M}(a, b, c, d):=\left\{(x, z, y) \mid(x+a, y+b) \in \operatorname{gph} S_{1},(y+c, z+d) \in \operatorname{gph} S_{2}\right\}
$$

at all points $((0,0,0,0),(\bar{x}, \bar{z}, \bar{y}))$ such that $\bar{y} \in \Xi(\bar{x}, \bar{z})$. Again, Proposition 2.6 and Remark 3.3 clarify that this assumption is equivalent to ours due to $M(b, d)=\widetilde{M}(0, b, 0, d)$ and $\widetilde{M}(a, b, c, d)=M(b-c, d)-(a, c, 0)$.

\subsection{Product Rule for Set-Valued Mappings}

Products of set-valued mappings are interesting on their own and also as an auxiliary tool to handle any binary operation on set-valued mappings, see [41, Section 3.2.3]. Arguably, the most common of such operations is addition. Here, we focus on the products. However, we also address the so-called decoupled sum rule along the way.

First, we investigate the simple case of componentwise products, where the set-valued mapping $S: \mathbb{R}^{n_{1}} \times \mathbb{R}^{n_{2}} \rightrightarrows \mathbb{R}^{\ell_{1}} \times \mathbb{R}^{\ell_{2}}$, given by

$$
S\left(y_{1}, y_{2}\right):=\Gamma_{1}\left(y_{1}\right) \times \Gamma_{2}\left(y_{2}\right)
$$

for mappings $\Gamma_{1}: \mathbb{R}^{n_{1}} \rightrightarrows \mathbb{R}^{\ell_{1}}$ and $\Gamma_{2}: \mathbb{R}^{n_{2}} \rightrightarrows \mathbb{R}^{\ell_{2}}$ with closed graphs, is under consideration. Since gph $S=\pi^{-1}\left(\operatorname{gph} \Gamma_{1} \times \operatorname{gph} \Gamma_{2}\right)$ holds, where $\pi\left(y_{1}, y_{2}, z_{1}, z_{2}\right):=\left(y_{1}, z_{1}, y_{2}, z_{2}\right)$ merely permutes the variables, derivatives of $S$ can be expressed as products of the derivatives associated with $\Gamma_{1}$ and $\Gamma_{2}$, see Lemmas 2.1 and 2.2. While for graphical derivatives and directional limiting coderivatives, we generally only get inclusions, equalities are obtained for the regular and the limiting coderivative. Furthermore, it is possible to get equalities for graphical derivative and directional limiting coderivative in particular situations where the use of Lemma 2.2 can be avoided, e.g. if one of the mappings $\Gamma_{1}$ or $\Gamma_{2}$ is single-valued and continuously differentiable. 
Let us investigate the setting $\ell:=\ell_{1}=\ell_{2}$. Composing the resulting mapping $S$ from (5.12) with the single-valued function sum: $\mathbb{R}^{\ell} \times \mathbb{R}^{\ell} \rightarrow \mathbb{R}^{\ell}$, given by $\operatorname{sum}\left(z_{1}, z_{2}\right):=z_{1}+z_{2}$, results in

$$
\Sigma\left(y_{1}, y_{2}\right):=(\operatorname{sum} \circ S)\left(y_{1}, y_{2}\right)=\Gamma_{1}\left(y_{1}\right)+\Gamma_{2}\left(y_{2}\right) .
$$

Thus, the generalized derivatives of $\Sigma: \mathbb{R}^{n_{1}} \times \mathbb{R}^{n_{2}} \rightrightarrows \mathbb{R}^{\ell}$ can be computed with the aid of the chain rule from Section 5.3. Continuous differentiability of sum implies (5.9) as well as calmness of the associated perturbation mapping (5.7) by (5.10). Hence, Theorem 5.2 yields the decoupled sum rule under the corresponding inner calmness* assumptions imposed on the intermediate mapping

$$
\Xi\left(\left(y_{1}, y_{2}\right), z\right)=\left\{\left(z_{1}, z_{2}\right) \in \Gamma_{1}\left(y_{1}\right) \times \Gamma_{2}\left(y_{2}\right) \mid z_{1}+z_{2}=z\right\} .
$$

These are, however, intrinsically satisfied if one of the mappings is single-valued and locally Lipschitzian. In particular, we exemplary get

$$
\begin{aligned}
D \Sigma\left(\left(\bar{y}_{1}, \bar{y}_{2}\right), \bar{z}\right)\left(v_{1}, v_{2}\right) & \subset D \Gamma_{1}\left(\bar{y}_{1}, \bar{z}-\gamma_{2}\left(\bar{y}_{2}\right)\right)\left(v_{1}\right)+D \gamma_{2}\left(\bar{y}_{2}\right)\left(v_{2}\right), \\
D^{*} \Sigma\left(\left(\bar{y}_{1}, \bar{y}_{2}\right), \bar{z}\right)\left(z^{*}\right) & \subset D^{*} \Gamma_{1}\left(\bar{y}_{1}, \bar{z}-\gamma_{2}\left(\bar{y}_{2}\right)\right)\left(z^{*}\right) \times D^{*} \gamma_{2}\left(\bar{y}_{2}\right)\left(z^{*}\right)
\end{aligned}
$$

if $\Gamma_{2}=\gamma_{2}$ holds for some single-valued, locally Lipschitzian function $\gamma_{2}: \mathbb{R}^{n_{2}} \rightarrow \mathbb{R}^{\ell}$. Moreover, if $\gamma_{2}$ is even continuously differentiable, all the estimates in fact hold with equality as well. This can be shown by Lemma 2.1 since we have gph $\Sigma=\left\{\left(\left(y_{1}, y_{2}\right), z\right) \mid\left(y_{1}, z-\right.\right.$ $\left.\left.\gamma_{2}\left(y_{2}\right)\right) \in \operatorname{gph} \Gamma_{1}\right\}$.

Next, we take a closer look at the setting $n:=n_{1}=n_{2}$. We want to compose the resulting mapping $S$ from (5.12) with the single-valued function doub: $\mathbb{R}^{n} \rightarrow \mathbb{R}^{n} \times \mathbb{R}^{n}$, given by $\operatorname{doub}(x):=(x, x)$, in order to compute the generalized derivatives of the product mapping $\Gamma: \mathbb{R}^{n} \rightrightarrows \mathbb{R}^{\ell_{1}} \times \mathbb{R}^{\ell_{2}}$ represented by

$$
\Gamma(x):=(S \circ \operatorname{doub})(x)=\Gamma_{1}(x) \times \Gamma_{2}(x) .
$$

Again, we are going to exploit the chain rule from Section 5.3 for these computations. Since doub is single-valued and continuously differentiable, (5.9) clearly holds, and, moreover, the associated intermediate mapping $\Xi$ from (5.6) is single- or empty-valued and, thus, trivially inner calm w.r.t. its domain at each point of its domain. On the other hand, the associated perturbation mapping $M: \mathbb{R}^{n} \times \mathbb{R}^{n} \times \mathbb{R}^{\ell_{1}} \times \mathbb{R}^{\ell_{2}} \rightrightarrows \mathbb{R}^{n} \times \mathbb{R}^{\ell_{1}} \times \mathbb{R}^{\ell_{2}} \times \mathbb{R}^{n} \times \mathbb{R}^{n}$ from (5.7) takes the form

$$
M\left(p_{1}, p_{2}, q_{1}, q_{2}\right)=\left\{\left(x, z_{1}, z_{2}, y_{1}, y_{2}\right) \mid x-y_{i}=p_{i}, q_{i} \in \Gamma_{i}\left(y_{i}\right)-z_{i}, i=1,2\right\} .
$$

Theorem 5.2 now yields the following result.

Theorem 5.5 Fix $\left(\bar{x},\left(\bar{z}_{1}, \bar{z}_{2}\right)\right) \in$ gph $\Gamma$. Then the following assertions hold.

D Graphical derivative: We always have

$$
D \Gamma\left(\bar{x},\left(\bar{z}_{1}, \bar{z}_{2}\right)\right)(u) \subset D \Gamma_{1}\left(\bar{x}, \bar{z}_{1}\right)(u) \times D \Gamma_{2}\left(\bar{x}, \bar{z}_{2}\right)(u),
$$

and the opposite inclusion holds if $M$ is calm at $\left((0,0,0,0),\left(\bar{x}, \bar{z}_{1}, \bar{z}_{2}, \bar{x}, \bar{x}\right)\right)$ and

$$
\begin{aligned}
& \left(\left(u, w_{1}\right),\left(u, w_{2}\right)\right) \in T_{\mathrm{gph} \Gamma_{1} \times \operatorname{gph} \Gamma_{2}}\left(\left(\bar{x}, \bar{z}_{1}\right),\left(\bar{x}, \bar{z}_{2}\right)\right) \\
& \Longleftrightarrow w_{i} \in D \Gamma_{i}\left(\bar{x}, \bar{z}_{i}\right)(u), i=1,2 .
\end{aligned}
$$

$\widehat{\mathbf{D}}^{*} \quad$ Regular coderivative: We always have

$$
\widehat{D}^{*} \Gamma\left(\bar{x},\left(\bar{z}_{1}, \bar{z}_{2}\right)\right)\left(z^{*}\right) \supset \widehat{D}^{*} \Gamma_{1}\left(\bar{x}, \bar{z}_{1}\right)\left(z_{1}^{*}\right)+\widehat{D}^{*} \Gamma_{2}\left(\bar{x}, \bar{z}_{2}\right)\left(z_{2}^{*}\right) .
$$


D $^{*} \quad$ Limiting coderivative: If $M$ is calm at $\left((0,0,0,0),\left(\bar{x}, \bar{z}_{1}, \bar{z}_{2}, \bar{x}, \bar{x}\right)\right)$, then

$$
D^{*} \Gamma\left(\bar{x},\left(\bar{z}_{1}, \bar{z}_{2}\right)\right)\left(z^{*}\right) \subset D^{*} \Gamma_{1}\left(\bar{x}, \bar{z}_{1}\right)\left(z_{1}^{*}\right)+D^{*} \Gamma_{2}\left(\bar{x}, \bar{z}_{2}\right)\left(z_{2}^{*}\right) .
$$

dD $^{*}$ Directional limiting coderivative: Fix a direction $\left(u, w_{1}, w_{2}\right) \in \mathbb{R}^{n} \times \mathbb{R}^{\ell_{1}} \times \mathbb{R}^{\ell_{2}}$. If $M$ is calm at $\left((0,0,0,0),\left(\bar{x}, \bar{z}_{1}, \bar{z}_{2}, \bar{x}, \bar{x}\right)\right)$ in direction $\left(u, w_{1}, w_{2}, u, u\right)$, then

$$
\begin{gathered}
D^{*} \Gamma\left(\left(\bar{x},\left(\bar{z}_{1}, \bar{z}_{2}\right)\right) ;\left(u,\left(w_{1}, w_{2}\right)\right)\right)\left(z^{*}\right) \\
\subset D^{*} \Gamma_{1}\left(\left(\bar{x}, \bar{z}_{1}\right) ;\left(u, w_{1}\right)\right)\left(z_{1}^{*}\right)+D^{*} \Gamma_{2}\left(\left(\bar{x}, \bar{z}_{2}\right) ;\left(u, w_{2}\right)\right)\left(z_{2}^{*}\right) .
\end{gathered}
$$

The qualification condition (5.10), which implies the calmness of $M$, takes the precise form

$$
D^{*} \Gamma_{1}\left(\bar{x}, \bar{z}_{1}\right)(0) \cap\left(-D^{*} \Gamma_{2}\left(\bar{x}, \bar{z}_{2}\right)(0)\right)=\{0\} .
$$

This is clearly satisfied if one of the mappings $\Gamma_{i}, i=1,2$, possesses the Aubin property at $\left(\bar{x}, \bar{z}_{i}\right)$. In the following lemma, we summarize some settings where (5.14) is naturally valid.

Lemma 5.6 Fix $\left(\bar{x},\left(\bar{z}_{1}, \bar{z}_{2}\right)\right) \in$ gph $\Gamma$. Then (5.14) holds under each of the following conditions:

(i) there are a locally Lipschitz continuous function $\gamma: \mathbb{R}^{n} \rightarrow \mathbb{R}^{\ell_{2}}$ as well as a closed set $\Omega \subset \mathbb{R}^{\ell_{2}}$ such that $\Gamma_{2}$ is given by $\Gamma_{2}(x):=\gamma(x)+\Omega$ (an analogous statements holds if $\Gamma_{1}$ admits such a representation);

(ii) the variables $x$ can be decomposed as $x=\left(x_{1}, x_{2}\right) \in \mathbb{R}^{n_{1}} \times \mathbb{R}^{n_{2}}$ and there exist setvalued mappings $\widetilde{\Gamma}_{1}: \mathbb{R}^{n_{1}} \rightrightarrows \mathbb{R}^{\ell_{1}}$ and $\widetilde{\Gamma}_{2}: \mathbb{R}^{n_{2}} \rightrightarrows \mathbb{R}^{\ell_{2}}$ as well as locally Lipschitz continuous functions $\gamma_{1}: \mathbb{R}^{n_{2}} \rightarrow \mathbb{R}^{\ell_{1}}$ and $\gamma_{2}: \mathbb{R}^{n_{1}} \rightarrow \mathbb{R}^{\ell_{2}}$ such that

$$
\Gamma_{1}(x):=\widetilde{\Gamma}_{1}\left(x_{1}\right)+\gamma_{1}\left(x_{2}\right) \quad \Gamma_{2}(x):=\widetilde{\Gamma}_{2}\left(x_{2}\right)+\gamma_{2}\left(x_{1}\right) .
$$

The estimates from Theorem 5.5 can be specified via the decoupled sum rule, see (5.13).

Proof In case (i), (5.14) follows from the Aubin property of $\Gamma_{2}$. Indeed, by the simple sum rule [41, Corollary 3.10], we obtain $D^{*} \Gamma_{2}\left(\bar{x}, \bar{z}_{2}\right)(0) \subset D^{*} \gamma(\bar{x})(0)$, where we exploited that $0 \in N_{\Omega}\left(\bar{z}_{2}-\gamma(\bar{x})\right)$ holds. Lipschitzness of $\gamma$ thus yields the claim. For the proof of (ii), (5.13) yields

$$
\begin{gathered}
D^{*} \Gamma_{1}\left(\left(\bar{x}_{1}, \bar{x}_{2}\right), \bar{z}_{1}\right)(0) \cap\left(-D^{*} \Gamma_{2}\left(\left(\bar{x}_{1}, \bar{x}_{2}\right), \bar{z}_{2}\right)(0)\right) \\
\subset\left(D^{*} \widetilde{\Gamma}_{1}\left(\bar{x}_{1}, \bar{z}_{1}\right)(0) \cap\left(-D^{*} \gamma_{2}\left(\bar{x}_{1}\right)(0)\right)\right) \times\left(D^{*} \gamma_{1}\left(\bar{x}_{2}\right)(0) \cap\left(-D^{*} \widetilde{\Gamma}_{2}\left(\bar{x}_{2}, \bar{z}_{2}\right)(0)\right)\right),
\end{gathered}
$$

and due to $D^{*} \gamma_{2}\left(\bar{x}_{1}\right)(0)=\{0\}$ as well as $D^{*} \gamma_{1}\left(\bar{x}_{2}\right)(0)=\{0\}$, which follows by local Lipschitz continuity of $\gamma_{2}$ and $\gamma_{1}$, we obtain validity of the constraint qualification (5.14).

In case where the single-valued functions appearing in the previous lemma are continuously differentiable, it is possible to obtain the product rule from Lemmas 2.1 and 2.2 which yields slightly stronger results.

Lemma 5.7 Fix $\left(\bar{x},\left(\bar{z}_{1}, \bar{z}_{2}\right)\right) \in \operatorname{gph} \Gamma$. Then the following assertions hold. 
(i) In the setting of Lemma 5.6 (i), if $\gamma$ is continuously differentiable, then, exemplary,

$$
\begin{aligned}
D \Gamma\left(\bar{x},\left(\bar{z}_{1}, \bar{z}_{2}\right)\right)(u) & \subset D \Gamma_{1}\left(\bar{x}, \bar{z}_{1}\right)(u) \times\left(\nabla \gamma(\bar{x}) u+T_{\Omega}\left(\bar{z}_{2}-\gamma(\bar{x})\right)\right), \\
D^{*} \Gamma\left(\bar{x},\left(\bar{z}_{1}, \bar{z}_{2}\right)\right)\left(z_{1}^{*}, z_{2}^{*}\right) & = \begin{cases}D^{*} \Gamma_{1}\left(\bar{x}, \bar{z}_{1}\right)\left(z_{1}^{*}\right)+\nabla \gamma(\bar{x})^{\top} z_{2}^{*} & -z_{2}^{*} \in N_{\Omega}\left(\bar{z}_{2}-\gamma(\bar{z})\right) \\
\varnothing & \text { otherwise, }\end{cases}
\end{aligned}
$$

and the analogous estimate for the regular coderivative also holds with equality.

(ii) In the setting of Lemma 5.6 (ii), if $\gamma_{1}$ and $\gamma_{2}$ are continuously differentiable, then we have equality in the formulas for the regular and limiting coderivative of $\Gamma$.

Proof We find a continuously differentiable function $g: \mathbb{R}^{n} \times \mathbb{R}^{\ell_{1}} \times \mathbb{R}^{\ell_{2}} \rightarrow \mathbb{R}^{n+\ell_{1}+\ell_{2}}$ with invertible Jacobian and a closed set $C \subset \mathbb{R}^{n+\ell_{1}+\ell_{2}}$ such that gph $\Gamma=g^{-1}(C)$ holds in both situations. More precisely, in (i), we can choose

$$
g\left(x, z_{1}, z_{2}\right):=\left(x, z_{1}, z_{2}-\gamma(x)\right) \quad C:=\operatorname{gph} \Gamma_{1} \times \Omega,
$$

while in (ii), we make use of

$$
g\left(\left(x_{1}, x_{2}\right), z_{1}, z_{2}\right):=\left(x_{1}, z_{1}-\gamma_{1}\left(x_{2}\right), x_{2}, z_{2}-\gamma_{2}\left(x_{1}\right)\right) \quad C:=\operatorname{gph} \Gamma_{1} \times \operatorname{gph} \Gamma_{2} .
$$

Thus, the results directly follow from Lemmas 2.1 and 2.2.

We want to finalize this section with a brief remark regarding the intersection rule from generalized differentiation.

Remark 5.8 Observe that we have

$$
\Gamma^{-1}\left(z_{1}, z_{2}\right)=\left\{x \mid z_{1} \in \Gamma_{1}(x), z_{2} \in \Gamma_{2}(x)\right\}=\Gamma_{1}^{-1}\left(z_{1}\right) \cap \Gamma_{2}^{-1}\left(z_{2}\right)
$$

by definition of $\Gamma$. Thus, we can rewrite the estimates and qualification conditions for products from Theorem 5.5 as well as Lemmas 5.6 and 5.7 in terms of intersections. Exemplary, let us mention that for some point $\left(\left(\bar{z}_{1}, \bar{z}_{2}\right), \bar{x}\right) \in \operatorname{gph} \Gamma^{-1}$ such that $M$ is calm at $\left((0,0,0,0),\left(\bar{x}, \bar{z}_{1}, \bar{z}_{2}, \bar{x}, \bar{x}\right)\right)$, we have

$$
\begin{aligned}
& D \Gamma^{-1}\left(\left(\bar{z}_{1}, \bar{z}_{2}\right), \bar{x}\right)\left(v_{1}, v_{2}\right) \quad \subset D \Gamma_{1}^{-1}\left(\bar{z}_{1}, \bar{x}\right)\left(v_{1}\right) \cap D \Gamma_{2}^{-1}\left(\bar{z}_{2}, \bar{x}\right)\left(v_{2}\right)
\end{aligned}
$$

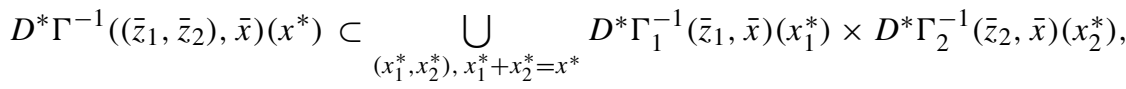

and similar estimates can be obtained for the regular and the directional limiting coderivative.

\section{Applications}

The importance of graphical derivative and limiting coderivative, in particular in connection with isolated calmness and the Aubin property, is well known, briefly mentioned in Section 1, and also demonstrated in the previous two sections. Well known is also the role of the limiting coderivative (limiting normal cone) in the formulation of reasonable first-order necessary optimality conditions for optimization problems, namely in form of so-called $M$-stationarity conditions. Here, we present some applications of the regular coderivative (regular subdifferential) and the directional limiting coderivative. 


\subsection{Optimality Conditions for Minimax Problems Based on Regular Subgradients}

Given a continuously differentiable function $\varphi: \mathbb{R}^{n} \times \mathbb{R}^{m} \rightarrow \mathbb{R}$ and a set-valued mapping $G: \mathbb{R}^{m} \rightrightarrows \mathbb{R}^{n}$ with closed graph, set

$$
f(x, y):=\varphi(x, y)+\delta_{\operatorname{gph} G^{-1}}(x, y) .
$$

Above, for some set $Q \subset \mathbb{R}^{\ell}, \delta_{Q}: \mathbb{R}^{\ell} \rightarrow \overline{\mathbb{R}}$ denotes the indicator function of $Q$ which vanishes on $Q$ and possesses value $\infty$ on $\mathbb{R}^{\ell} \backslash Q$. Clearly, $f$ is a lower semicontinuous function due to epi $f=\operatorname{epi} \varphi \cap\left(\mathrm{gph} G^{-1} \times \mathbb{R}\right)$.

We consider the following optimization problem of minimax type

$$
\max _{y \in \Omega} \min _{x \in G(y)} \varphi(x, y)
$$

(MaxMin)

where $\Omega \subset \mathbb{R}^{m}$ is a closed set. Problems of this type arise frequently in game theory and can be interpreted as a particular instance of bilevel optimization. We refer the interested reader to $[10,12]$ for an introduction to minimax programming and to the monographs $[11$, 41] for a detailed discussion of bilevel optimization.

Using the function $f$ defined above and the associated marginal function $\vartheta: \mathbb{R}^{m} \rightarrow \overline{\mathbb{R}}$ from Section 5.2, we easily see that

$$
\min _{y \in \Omega}(-\vartheta)(y)
$$

is a suitable surrogate problem of (MaxMin). Subsequently, we exploit $S: \mathbb{R}^{m} \rightrightarrows \mathbb{R}^{n}$ in order to denote the solution mapping of the inner minimization problem $\min _{x}\{\varphi(x, y) \mid x \in$ $G(y)\}$. Furthermore, we make use of the mapping $M: \mathbb{R}^{m} \times \mathbb{R} \rightrightarrows \mathbb{R}^{n}$ given by

$$
M(y, \alpha):=\{x \in G(y) \mid(x, y, \alpha) \in \text { epi } \varphi\} .
$$

Theorem 6.1 Let $(\bar{x}, \bar{y}) \in \mathbb{R}^{n} \times \mathbb{R}^{m}$ be a locally optimal solution of (MaxMin), i.e., assume that there is a neighborhood $V$ of $\bar{y}$ such that $\vartheta(\bar{y}) \geq \vartheta(y)$ holds for all $y \in \Omega \cap V$ while $\bar{x} \in S(\bar{y})$ is valid. Furthermore, let $M$ be inner calm* at $(\bar{y}, \vartheta(\bar{y}))$ w.r.t. $\operatorname{dom} M$ in the fuzzy sense. Then the following condition holds:

$$
\left[y^{*} \in \nabla_{y} \varphi(x, \bar{y})+\widehat{D}^{*} G(\bar{y}, x)\left(\nabla_{x} \varphi(x, \bar{y})\right) \forall x \in S(\bar{y})\right] \Longrightarrow y^{*} \in \widehat{N}_{\Omega}(\bar{y}) .
$$

Proof The assumptions of the theorem guarantee that $\bar{y}$ is a local minimizer of (6.1). Thus, [42, Theorem 4.3(i)] guarantees validity of

$$
\widehat{\partial} \vartheta(\bar{y}) \subset \widehat{N}_{\Omega}(\bar{y}) \text {. }
$$

Due to the fuzzy inner calmness* of $M$, Theorem 5.1 guarantees

$$
\begin{gathered}
\widehat{\partial} \vartheta(\bar{y}) \quad\left\{y^{*} \mid\left(0, y^{*}\right) \in \bigcap_{x \in S(\bar{y})} \widehat{\partial} f(x, \bar{y})\right\} \\
=\left\{y^{*} \mid\left(0, y^{*}\right) \in \bigcap_{x \in S(\bar{y})}\left(\nabla \varphi(x, \bar{y})+\widehat{N}_{\mathrm{gph} G^{-1}}(x, \bar{y})\right)\right\}
\end{gathered}
$$

where we used the sum rule from [42, Proposition 2.2] in order to decompose the regular subdifferential of $f$, and the equivalence of regular subgradients of the indicator function associated with a locally closed set as well as its regular normals, see [46, Exercise 8.14]. Elementary calculations now show

$$
y^{*} \in \widehat{\partial} \vartheta(\bar{y}) \quad \Longleftrightarrow \quad\left(y^{*}-\nabla_{y} \varphi(x, \bar{y}),-\nabla_{x} \varphi(x, \bar{y})\right) \in \widehat{N}_{\mathrm{gph} G}(\bar{y}, x) \quad \forall x \in S(\bar{y}) .
$$

Exploiting the definition of the regular coderivative as well as (6.2), the result follows. 
Invoking Corollary 4.9 and (5.5) as well as the calculus rules for the directional limiting subdifferential from [6], we observe that $M$ is inner calm* in the fuzzy sense w.r.t. dom $M$ at $(\bar{y}, \vartheta(\bar{y}))$ whenever it is inner semicompact there while the condition

$$
\begin{gathered}
\forall u \in D G(\bar{y}, x)(0) \backslash\{0\}: \\
\nabla_{x} \varphi(x, \bar{y}) u \leq 0 \Longrightarrow \nabla_{y} \varphi(x, \bar{y})+D^{*} G((\bar{y}, x) ;(0, u))\left(\nabla_{x} \varphi(x, \bar{y})\right) \subset\{0\}
\end{gathered}
$$

holds for each $x \in S(\bar{y})$.

In order to infer a necessary optimality condition in terms of initial problem data from (6.2), it is indeed necessary to find a reasonable lower estimate for $\widehat{\partial} \vartheta(\bar{y})$. Here, our novel results from Theorem 5.1, which yield an equivalent representation of this subdifferential and, thus, an optimality condition of reasonable strength, work quite nicely. In practice, it may happen that $\widehat{\partial} \vartheta(\bar{y})$ is empty for points $(\bar{x}, \bar{y})$ which are locally optimal to (MaxMin), and in this case, (6.2) holds trivially. However, one could still use Theorem 6.1 in order to exclude points which do not provide locally optimal solutions of (MaxMin) in this case.

In order to infer necessary optimality conditions in terms of limiting normals for (MaxMin), one could presume local Lipschitzness of $\vartheta$ around the point of interest $\bar{y}$ so that [46, Theorem 8.15] yields $0 \in \partial(-\vartheta)(\bar{y})+N_{\Omega}(\bar{y})$. Now, to apply our results from Theorem 5.1, we need to convexify the limiting subdifferential in order to get rid of the negative sign, i.e., we have to evaluate $0 \in-\operatorname{conv} \partial \vartheta(\bar{y})+N_{\Omega}(\bar{y})$ which is possible now with the aid of Carathéodory's theorem whenever $M$ from above is inner semicompact at $(\bar{y}, \vartheta(\bar{y}))$.

\subsection{Semismoothness* Calculus}

In the recent paper [20], a new notion of semismoothness $*$ for sets and set-valued mappings has been introduced and used to propose a semismooth* Newton method for solving generalized equations. This notion is based on the directional limiting constructions and so our results yield calculus rules for semismoothness*, i.e., the rules that describe how this property can be transferred from one or more objects to another by the transformations discussed in Section 5.

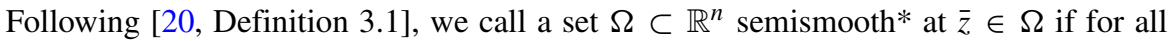
$w \in \mathbb{R}^{n}$, it holds

$$
\left\langle z^{*}, w\right\rangle=0 \quad \forall z^{*} \in N_{\Omega}(\bar{z} ; w) .
$$

A set-valued mapping $M: \mathbb{R}^{m} \rightrightarrows \mathbb{R}^{n}$ is called semismooth* at $(\bar{y}, \bar{x}) \in \operatorname{gph} M$ if $\operatorname{gph} M$ is semismooth* at $(\bar{y}, \bar{x})$, i.e., for all $(v, u) \in \mathbb{R}^{m} \times \mathbb{R}^{n}$, we have

$$
\left\langle y^{*}, v\right\rangle=\left\langle x^{*}, u\right\rangle \quad \forall\left(x^{*}, y^{*}\right) \in \operatorname{gph} D^{*} M((\bar{y}, \bar{x}) ;(v, u)) .
$$

Theorem 6.2 Let $M: \mathbb{R}^{m} \rightrightarrows \mathbb{R}^{n}$ be a set-valued mapping.

(i) Suppose that $\bar{y} \in \operatorname{dom} M$ is chosen such that gph $M$ is locally closed around $\{\bar{y}\} \times \mathbb{R}^{n}$ and $\operatorname{dom} M$ is locally closed around $\bar{y}$. If $M$ is semismooth* at $(\bar{y}, \bar{x})$ for each $\bar{x} \in$ $M(\bar{y})$ as well as inner calm* at $\bar{y}$ w.r.t. $\operatorname{dom} M$, then $\operatorname{dom} M$ is semismooth* at $\bar{y}$.

(ii) Suppose that $M$ is locally closed around $(\bar{y}, \bar{x}) \in \operatorname{gph} M$. If $M$ is semismooth* as well as calm at $(\bar{y}, \bar{x})$, then $M(\bar{y})$ is semismooth* at $\bar{x}$.

Proof To show (i), pick $v \in \mathbb{R}^{m}$ and $y^{*} \in N_{\mathrm{dom} M}(\bar{y} ; v)$. By Theorem 3.1, we infer that there exist $\bar{x} \in M(\bar{y})$ and $u \in D M(\bar{y}, \bar{x})(v)$ such that $y^{*} \in D^{*} M((\bar{y}, \bar{x}) ;(v, u))(0)$ holds. Semismoothness $*$ of $M$ at $(\bar{y}, \bar{x})$ now readily yields $\left\langle y^{*}, v\right\rangle=\langle 0, u\rangle=0$. 
To show (ii), let $u \in \mathbb{R}^{n}$ and $x^{*} \in N_{M(\bar{y})}(\bar{x} ; u)$ be given. Theorem 3.2 implies the existence of $y^{*} \in D^{*} M((\bar{y}, \bar{x}) ;(0, u))\left(-x^{*}\right)$ and, hence, from semismoothness* of $M$ at $(\bar{y}, \bar{x})$, we get $\left\langle x^{*}, u\right\rangle=-\left\langle y^{*}, 0\right\rangle=0$.

Naturally, one can derive similar results in more useful situations described in Section 5. That is to say, in the settings of standard calculus rules, semismoothness* can be transferred to the desired object not only from $M$, but also from (semismoothness* of) the input data. Exemplary, semismoothness* of the image representation mapping $M_{3}(y):=g^{-1}(y) \cap C$, see Section 5.1, is implied by semismoothness* of $g: \mathbb{R}^{n} \rightarrow \mathbb{R}^{n}$ and $C \subset \mathbb{R}^{n}$, provided $g$ is Lipschitz continuous. Hence, semismoothness* is transferred from $g$ and $C$, via $M_{3}$, to $g(C)=\operatorname{dom} M_{3}$.

\section{Concluding Remarks}

In this paper, we have seen that the two enhanced estimates stated in Theorems 3.1 and 3.2 are enough to recover the major calculus rules of variational analysis regarding tangents as well as regular, limiting, and directional limiting normals under mild calmness-type conditions. As a by-product, we obtained calculus rules for the computation of generalized derivatives associated with Cartesian products of set-valued mappings in Section 5.4. In Section 4, we interrelated the different (inner) calmness-type conditions which were used to establish the two patterns from Theorems 3.1 and 3.2. Particularly, we have shown that Gfrerer's first-order sufficient condition for calmness can be used to guarantee the validity of inner calmness* in the fuzzy sense. It remains an open question whether the former condition already yields inner calmness*. Furthermore, a precise study on the different moduli of the calmness-type properties seems to be an interesting subject of future research.

Acknowledgments The research of the first author was supported by the Austrian Science Fund (FWF) under grants P29190-N32 and P32832-N. The authors are grateful to Jiří V. Outrata, who suggested the application to semismoothness* from Section 6.2.

Funding Open access funding provided by University of Vienna.

Open Access This article is licensed under a Creative Commons Attribution 4.0 International License, which permits use, sharing, adaptation, distribution and reproduction in any medium or format, as long as you give appropriate credit to the original author(s) and the source, provide a link to the Creative Commons licence, and indicate if changes were made. The images or other third party material in this article are included in the article's Creative Commons licence, unless indicated otherwise in a credit line to the material. If material is not included in the article's Creative Commons licence and your intended use is not permitted by statutory regulation or exceeds the permitted use, you will need to obtain permission directly from the copyright holder. To view a copy of this licence, visit http://creativecommons.org/licenses/by/4.0/.

\section{References}

1. Adam, L., Henrion, R., Outrata, J.: On M-stationarity conditions in MPECs and the associated qualification conditions. Math. Program. 168(1), 229-259 (2018)

2. Aubin, J.-P., Ekeland, I.: Applied Nonlinear Analysis. Wiley-Interscience, New York (1984)

3. Aubin, J.-P., Frankowska, H.: Set-Valued Analysis. Birkhäuser, Boston (2009)

4. Benko, M.: On inner calmness*, generalized calculus, and derivatives of the normal cone mapping. arXiv pages 1-27 (2019) 
5. Benko, M., Červinka, M., Hoheisel, T.: Sufficient conditions for metric subregularity of constraint systems with applications to disjunctive and ortho-disjunctive programs. Set-Valued Var. Anal., 1-35 (2021)

6. Benko, M., Gfrerer, H., Outrata, J.V.: Calculus for directional limiting normal cones and subdifferentials. Set-Valued Var. Anal. 27(3), 713-745 (2019)

7. Benko, M., Gfrerer, H., Outrata, J.V.: Stability analysis for parameterized variational systems with implicit constraints. Set-Valued Var. Anal. 28(1), 167-193 (2020)

8. Cibulka, R., Fabian, M., Kruger, A.Y.: On semiregularity of mappings. J. Math. Anal. Appl. 473(2), 811-836 (2019)

9. Cánovas, M., Gisbert, M., Henrion, R., Parra, J.: Lipschitz lower semicontinuity moduli for linear inequality systems. J. Math. Anal. Appl. 490(2), 1-21 (2020)

10. Danskin, J.M.: The theory of max-min, with applications. SIAM J. Appl. Math. 14(4), 641-664 (1966)

11. Dempe, S.: Foundations of Bilevel Programming. Kluwer, Dordrecht (2002)

12. Demyanov, V.F., Malozemov, V.V.: Introduction to Minimax. Wiley, New York (1974)

13. Dontchev, A.L., Rockafellar, R.T.: Regularity and conditioning of solution mappings in variational analysis. Set-Valued Anal. 12, 79-109 (2004)

14. Dontchev, A.L., Rockafellar, R.T.: Implicit Functions and Solution Mappings. Springer, Heidelberg (2014)

15. Fabian, M.J., Henrion, R., Kruger, A.Y., Outrata, J.V.: Error bounds: necessary and sufficient conditions. Set-Valued Var. Anal. 18(2), 121-149 (2010)

16. Gfrerer, H.: On directional metric regularity, subregularity and optimality conditions for nonsmooth mathematical programs. Set-Valued Var. Anal. 21(2), 151-176 (2013)

17. Gfrerer, H.: Optimality conditions for disjunctive programs based on generalized differentiation with application to mathematical programs with equilibrium constraints. SIAM J. Optim. 24(2), 898-931 (2014)

18. Gfrerer, H., Klatte, D.: Lipschitz and Hölder stability of optimization problems and generalized equations. Math. Program. 158, 35-75 (2016)

19. Gfrerer, H., Outrata, J.V.: On Lipschitzian properties of implicit multifunctions. SIAM J. Optim. 26(4), 2160-2189 (2016)

20. Gfrerer, H., Outrata, J.V.: On a semismooth* Newton method for solving generalized equations. SIAM J. Optim. 31(1), 489-517 (2021)

21. Gfrerer, H., Ye, J.J.: New constraint qualifications for mathematical programs with equilibrium constraints via variational analysis. SIAM J. Optim. 27(2), 842-865 (2017)

22. Gfrerer, H., Ye, J.J.: New sharp necessary optimality conditions for mathematical programs with equilibrium constraints. Set-Valued Var. Anal. 28(2), 395-426 (2020)

23. Ginchev, I., Mordukhovich, B.S.: On directionally dependent subdifferentials. Proc. Bulgarian Acad. Sci. 64(4), 497-508 (2011)

24. Harder, F., Wachsmuth, G.: The limiting normal cone of a complementarity set in Sobolev spaces. Optimization 67(10), 1579-1603 (2018)

25. Henrion, R., Jourani, A., Outrata, J.V.: On the calmness of a class of multifunctions. SIAM J. Optim. 13(2), 603-618 (2002)

26. Henrion, R., Outrata, J.V.: Calmness of constraint systems with applications. Math. Program. 104(1), 437-464 (2005)

27. Ioffe, A.D.: Necessary and sufficient conditions for a local minimum. 1: A reduction theorem and first order conditions. SIAM J. Control. Optim. 17(2), 245-250 (1979)

28. Ioffe, A.D.: Variational Analysis of Regular Mappings. Springer, Cham (2017)

29. Ioffe, A.D., Outrata, J.V.: On metric and calmness qualification conditions in subdifferential calculus. Set-Valued Anal. 16(2), 199-227 (2008)

30. Ioffe, A.D., Penot, J.-P.: Subdifferentials of performance functions and calculus of coderivatives of setvalued mappings. Serdica Math. J. 22(3), 359-384 (1996)

31. Klatte, D.: A note on quantitative stability results in nonlinear programming. Seminarbericht, Sektion Mathematik. Humboldt-Universität zu Berlin, 90, 77-86 (1987)

32. Klatte, D.: On quantitative stability for non-isolated minima. Control. Cybern. 23, 183-200 (1994)

33. Klatte, D., Kummer, B.: Nonsmooth Equations in Optimization. Kluwer Academic, Dordrecht (2002)

34. Klatte, D., Kummer, B.: On calmness of the argmin mapping in parametric optimization problems. J. Optim. Theory Appl. 165(3), 708-719 (2015)

35. Levy, A.B.: Implicit multifunction theorems for the sensitivity analysis of variational conditons. Math. Program. 74, 333-350 (1996)

36. Long, P., Wang, B., Yang, X.: Calculus for directional coderivatives and normal cones in Asplund spaces. Positivity 21, 1115-1142 (2017) 
37. Mehlitz, P.: On the sequential normal compactness condition and its restrictiveness in selected function spaces. Set-Valued Var. Anal. 27(3), 763-782 (2019)

38. Mehlitz, P., Wachsmuth, G.: The limiting normal cone to pointwise defined sets in Lebesgue spaces. Set-Valued Var. Anal. 26(3), 449-467 (2018)

39. Mordukhovich, B.S.: Maximum principle in the problem of time optimal response with nonsmooth constraints. J. Appl. Math. Mech. 40(6), 960-969 (1976)

40. Mordukhovich, B.S.: Variational Analysis and Generalized Differentiation, Vol. I: Basic Theory, Vol. II: Applications. Springer, Berlin (2006)

41. Mordukhovich, B.S.: Variational Analysis and Applications. Springer, Cham (2018)

42. Mordukhovich, B.S., Nam, N.M., Yen, N.D.: Frèchet subdifferential calculus and optimality conditions in nondifferentiable programming. Optimization 55(5-6), 685-708 (2006)

43. Nechita, D.-M.: About some links between the Dini-Hadamard-like normal cone and the contingent cone. Stud. Univ. Babes-Bolyai Math. 57(4), 541-549 (2012)

44. Robinson, S.M.: Some continuity properties of polyhedral multifunctions. In: König, H., Korte, B., Ritter, K. (eds.) Mathematical Programming at Oberwolfach, pp. 206-214. Springer, Berlin (1981)

45. Rockafellar, R.T.: Convex Analysis. Princeton University Press, Princeton (1970)

46. Rockafellar, R.T., Wets, R.J.-B.: Variational Analysis. Springer, Berlin (1998)

47. Ye, J.J., Zhou, J.: Verifiable sufficient conditions for the error bound property of second-order cone complementarity problems. Math. Program. 171(1), 361-395 (2018)

Publisher's Note Springer Nature remains neutral with regard to jurisdictional claims in published maps and institutional affiliations. 